\title{
Gradient Estimates and Harnack Inequality for a Nonlinear Parabolic Equation on Complete Manifolds
}

\author{
Jiaxian Wu • Yi-Hu Yang
}

Received: 26 November 2013 / Revised: 30 January 2014 / Accepted: 10 February 2014 /

Published online: 21 March 2014

(C) School of Mathematical Sciences, University of Science and Technology of China and Springer-Verlag Berlin Heidelberg 2014

Abstract Let $M$ be a noncompact complete Riemannian manifold. In this paper, we consider the following nonlinear parabolic equation on $M$

$$
u_{t}(x, t)=\Delta u(x, t)+a u(x, t) \ln u(x, t)+b u^{\alpha}(x, t) .
$$

We prove a Li-Yau type gradient estimate for positive solutions to the above equation; as an application, we also derive the corresponding Harnack inequality. These results generalize the corresponding ones proved by Li (J Funct Anal 100:233-256, 1991).

Keywords Gradient estimate $\cdot$ Ricci curvature $\cdot$ Harnack inequality · Nonlinear parabolic equation

Mathematics Subject Classification (2010) $\quad 58 \mathrm{~J} 35 \cdot 35 \mathrm{~K} 10 \cdot 35 \mathrm{~K} 55$

\section{Introduction}

Let $M$ be an $n$-dimensional Riemannian manifold with nonnegative Ricci curvature, in particular, the $n$-dimensional Euclidean space. In [3], Gidas and Spruck studied the following elliptic equation on $M$

$$
\Delta u+u^{\alpha}=0
$$

\footnotetext{
J. Wu

Department of Mathematics, Tongji University, Shanghai 200092, China e-mail: jiaxian.wu@gmail.com

Y.-H. Yang $(\bowtie)$

Department of Mathematics, Shanghai Jiao Tong University, Shanghai 200240, China

e-mail: yangyihu@sjtu.edu.cn
} 
where $1 \leq \alpha \leq \frac{n+2}{n-2}$. They showed that any nonnegative solution of (1.1) have to be zero.

A natural question is what the situation is for the corresponding parabolic equation of (1.1). In [6], Li, by generalized the results of [5], considered this question. More precisely, he studied the following parabolic equation

$$
u_{t}(x, t)=\Delta u(x, t)+h(x, t) u^{\alpha}(x, t),
$$

on $M \times[0, \infty)$, where $\alpha$ is a positive constant and $h(x, t)$ is a function defined on $M \times[0, \infty)$, which is $C^{2}$ in the $x$-variable and $C^{1}$ in the $t$-variable. His main result can be stated as follows:

Theorem A Let $\left(M, g=g_{i j}\right)$ be an n-dimensional complete Riemannian manifold with Ricci tensor $R_{i j} \geq-k g_{i j}(k \geq 0)$. Let $h(x, t)$ be a nonnegative function defined on $M \times[0, \infty)$, which is $C^{2}$ in the $x$-variable and $C^{1}$ in the $t$-variable. Assume that $0<\alpha<n /(n-1)$ and $(\Delta+\partial / \partial t) h(x, t) \geq 0$. If $u(x, t)$ is a positive solution of (1.2), then

$$
\frac{|\nabla u|^{2}}{u^{2}}+\frac{h u^{\alpha-1}}{\alpha}-\frac{1}{\alpha} \frac{u_{t}}{u} \leq \frac{1}{t} \frac{2 n}{\alpha^{2}}+\frac{2 n K}{(2-\alpha) \alpha}
$$

and

$$
u\left(x_{1}, t_{1}\right) \leq u\left(x_{2}, t_{2}\right)\left(\frac{t_{2}}{t_{1}}\right)^{\frac{2 n}{\alpha}} \exp \left(\frac{\rho^{2}}{4 \alpha\left(t_{2}-t_{1}\right)}+\frac{\left(t_{2}-t_{1}\right) 2 n K}{(2-\alpha)}\right)
$$

where $x_{1}, x_{2} \in M, 0<t_{1}<t_{2}<\infty$, and $\rho\left(x_{1}, x_{2}\right)$ is the geodesic distance between $x_{1}$ and $x_{2}$.

On the other hand, to understand the gradient Ricci soliton [4], Ma [7] studied the following elliptic equation

$$
\Delta u(x)+a u(x) \ln u(x)+b u(x)=0,
$$

where $a$ and $b$ are constants and $a<0$. The corresponding parabolic equation of (1.5) was studied by Yang [8] and he also got a Li-Yau type gradient estimate.

In this paper, we want to study the following parabolic equation

$$
u_{t}(x, t)=\Delta u(x, t)+a u(x, t) \ln u(x, t)+b u^{\alpha}(x, t)
$$

on $M \times[0, T](T>0)$, where $a$ and $b$ are real constants and $\alpha>0$. Our purpose is to generalize the above result of $\mathrm{Li}$ to this case to obtain some gradient estimates of Li-Yau type. The main result can be stated as follows:

Theorem B Let $M$ be an n-dimensional complete noncompact Riemannian manifold with Ricci curvature bounded below by $-K(K \geq 0)$. Assume $0<\alpha<\frac{n}{n-1}$ and a 
and $b$ in (1.6) are nonnegative constants. If $u(x, t)$ is a positive solution of (1.6) on $M \times[0, T]$ and $\ln u \geq 0$, then we have the following gradient estimate for $t>0$

$$
\begin{aligned}
& \frac{|\nabla u|^{2}}{u^{2}}+\frac{b}{\alpha} u^{\alpha-1}+\frac{a}{\alpha} \ln u-\frac{2}{\alpha} \frac{u_{t}}{u} \leq \frac{1}{t} \frac{2 n}{\alpha^{2}}+\frac{2 n a}{\alpha}+\frac{2 n K}{(2-\alpha) \alpha} \\
& +\frac{n a}{\alpha(2-\alpha)}\left|\frac{1}{\alpha}-1\right| .
\end{aligned}
$$

Consequently, the following Harnack type inequality

$$
\begin{aligned}
u\left(x_{1}, t_{1}\right) \leq & u\left(x_{2}, t_{2}\right)\left(\frac{t_{2}}{t_{1}}\right)^{\frac{n}{\alpha}} \exp \left(\frac{\rho^{2}}{2\left(t_{2}-t_{1}\right) \alpha}+\frac{\left(t_{2}-t_{1}\right) n K}{(2-\alpha)}\right. \\
& \left.+n a\left(t_{2}-t_{1}\right)+\frac{n a}{2(2-\alpha)}\left|\frac{1}{\alpha}-1\right|\left(t_{2}-t_{1}\right)\right)
\end{aligned}
$$

holds, where $x_{1}, x_{2} \in M, 0<t_{1}<t_{2}<T$, and $\rho\left(x_{1}, x_{2}\right)$ is the geodesic distance between $x_{1}$ and $x_{2}$.

Remarks In Theorem B, if $a=0$, the condition $\ln u \geq 0$ is unnecessary. In this case, (1.7) and (1.8) can be written as follows:

$$
\frac{|\nabla u|^{2}}{u^{2}}+\frac{b}{\alpha} u^{\alpha-1}-\frac{2}{\alpha} \frac{u_{t}}{u} \leq \frac{1}{t} \frac{2 n}{\alpha^{2}}+\frac{2 n K}{(2-\alpha) \alpha}
$$

and

$$
u\left(x_{1}, t_{1}\right) \leq u\left(x_{2}, t_{2}\right)\left(\frac{t_{2}}{t_{1}}\right)^{\frac{n}{\alpha}} \exp \left(\frac{\rho^{2}}{2\left(t_{2}-t_{1}\right) \alpha}+\frac{\left(t_{2}-t_{1}\right) n K}{(2-\alpha)}\right)
$$

which are similar to (1.3) and (1.4) (see also Theorem 2.3 in Li [6]). When $h(x, t)$ in (1.2) is positive constant, then the two inequalities above are essentially equivalent to (1.3) and (1.4), respectively.

This paper is organized as follows: In Sect. 2, we will prove two preliminary lemmas, which are key to show our gradient estimates for (1.6), while the gradient estimates are presented in Sect. 3. As an application of the gradient estimate, in the final section, we prove Harnack inequality.

\section{Two Preliminary Lemmas}

In this section, our purpose is to show two lemmas, which will play a key role in the proof of the gradient estimate. Suppose that $u(x, t)$ is a positive solution of (1.6). We define

$$
W(x, t)=(u(x, t))^{-\beta},
$$


where $\beta$ is a positive constant to be determined. A direct computation shows

$$
\begin{aligned}
\frac{|\nabla W|^{2}}{W^{2}} & =\beta^{2} \frac{|\nabla u|^{2}}{u^{2}}, \\
\frac{W_{t}}{W} & =-\beta \frac{u_{t}}{u},
\end{aligned}
$$

and

$$
\begin{aligned}
\Delta W & =\beta(\beta+1) u^{-\beta-2}|\nabla u|^{2}-\beta u^{-\beta-1} \Delta u \\
& =\beta(\beta+1) W \frac{|\nabla u|^{2}}{u^{2}}-\beta u^{-\beta-1}\left(u_{t}-a u \ln u-b u^{\alpha}\right) \\
& =\frac{\beta+1}{\beta} \frac{|\nabla W|^{2}}{W}-\beta u^{-\beta-1} u_{t}+\beta a u^{-\beta} \ln u+\beta b u^{\alpha-\beta-1} \\
& =\frac{\beta+1}{\beta} \frac{|\nabla W|^{2}}{W}+W_{t}-a W \ln W+\beta b W^{1+\frac{1-\alpha}{\beta}} .
\end{aligned}
$$

Therefore,

$$
\left(\Delta-\frac{\partial}{\partial t}\right) W=\frac{\beta+1}{\beta} \frac{|\nabla W|^{2}}{W}-a W \ln W+\beta b W^{1+\frac{1-\alpha}{\beta}} .
$$

Set

$$
\begin{aligned}
G_{0} & =\frac{|\nabla W|^{2}}{W^{2}}+b \nu W^{\frac{1-\alpha}{\beta}}-\gamma \ln W, \\
G_{1} & =\frac{W_{t}}{W}, \\
G & =G_{0}+\delta G_{1},
\end{aligned}
$$

where $\nu, \gamma$, and $\delta$ are some positive constants to be fixed.

Let $\left\{e_{1}, e_{2}, \ldots, e_{n}\right\}$ be a local orthonormal frame field on $M$. We use the convention that the subscripts $i, j, k, \ldots(1 \leq i, j, k, \ldots \leq n)$ mean the covariant derivatives in the $e_{i}, e_{j}, e_{k}, \ldots$ directions, respectively; namely, we write $\nabla_{e_{i}} W, \nabla_{e_{j}} \nabla_{e_{i}} W, \nabla_{e_{k}} \nabla_{e_{j}} \nabla_{e_{i}} W, \ldots$ briefly as $W_{i}, W_{i j}, W_{i j k}, \ldots$, respectively. $R_{i j}$ is the Ricci tensor of $M$.

A simple computation gives

$$
\begin{aligned}
\nabla G_{1} & =\frac{W_{t i}}{W}-\frac{W_{t} W_{i}}{W^{2}}, \\
\Delta G_{1} & =\frac{W_{t i i}}{W}-2 \frac{W_{t i} W_{i}}{W^{2}}-\frac{W_{t} W_{i i}}{W^{2}}+2 \frac{W_{t} W_{i}^{2}}{W^{3}}, \\
\frac{\partial}{\partial t} G_{1} & =\frac{W_{t t}}{W}-\frac{W_{t}^{2}}{W^{2}},
\end{aligned}
$$


so, we have

$$
\begin{aligned}
\left(\Delta-\frac{\partial}{\partial t}\right) G_{1} & =\frac{W_{t i i}}{W}-2 \frac{W_{t i} W_{i}}{W^{2}}-\frac{W_{t} W_{i i}}{W^{2}}+2 \frac{W_{t} W_{i}^{2}}{W^{3}}-\frac{W_{t t}}{W}+\frac{W_{t}^{2}}{W^{2}} \\
& =\frac{\left(W_{i i}-W_{t}\right)_{t}}{W}-\frac{\left(\Delta W-W_{t}\right) W_{t}}{W^{2}}-2\left(\frac{W_{t i}}{W}-\frac{W_{t} W_{i}}{W^{2}}\right) \frac{W_{i}}{W} \\
& =\left(\frac{\Delta W-W_{t}}{W}\right)_{t}-2\left(\frac{W_{t i}}{W}-\frac{W_{t} W_{i}}{W^{2}}\right) \frac{W_{i}}{W} .
\end{aligned}
$$

Substituting (2.2) into the above equality, one has

$$
\begin{aligned}
(\Delta & \left.-\frac{\partial}{\partial t}\right) G_{1} \\
= & \left(\frac{\beta+1}{\beta} \frac{|\nabla W|^{2}}{W^{2}}-a \ln W+\beta b W^{\frac{1-\alpha}{\beta}}\right)_{t}-2\left(\frac{W_{t i}}{W}-\frac{W_{t} W_{i}}{W^{2}}\right) \frac{W_{i}}{W} \\
= & 2 \frac{\beta+1}{\beta} \frac{W_{i t} W_{i}}{W^{2}}-2 \frac{\beta+1}{\beta} \frac{|\nabla W|^{2} W_{t}}{W^{3}}-a \frac{W_{t}}{W} \\
& +b(1-\alpha) W^{\frac{1-\alpha}{\beta}-1} W_{t}-2\left(\frac{W_{i t}}{W}-\frac{W_{i} W_{t}}{W^{2}}\right) \frac{W_{i}}{W} \\
= & 2\left(\frac{\beta+1}{\beta}-1\right)\left(\frac{W_{i t}}{W}-\frac{W_{i} W_{t}}{W^{2}}\right) \frac{W_{i}}{W}-a \frac{W_{t}}{W}+b(1-\alpha) W^{\frac{1-\alpha}{\beta}} \frac{W_{t}}{W} \\
= & \frac{2}{\beta} \nabla G_{1} \cdot \nabla \ln W-a \frac{W_{t}}{W}+b(1-\alpha) W^{\frac{1-\alpha}{\beta}} \frac{W_{t}}{W} .
\end{aligned}
$$

On the other hand, we also have

$$
\begin{aligned}
\nabla G_{0}= & \frac{2 W_{i} W_{i j}}{W^{2}}-\frac{2|\nabla W|^{2} W_{j}}{W^{3}}+\frac{b v(1-\alpha) W_{j} W^{\frac{1-\alpha}{\beta}-1}}{\beta}-\gamma \frac{W_{j}}{W}, \\
\Delta G_{0}= & \frac{2 W_{i j}^{2}}{W^{2}}+\frac{2 W_{i} W_{i j j}}{W^{2}}-\frac{4 W_{i} W_{j} W_{i j}}{W^{3}}-\frac{4 W_{i j} W_{i} W_{j}}{W^{3}}-\frac{2|\nabla W|^{2} W_{j j}}{W^{3}} \\
& +\frac{6|\nabla W|^{2} W_{j}^{2}}{W^{4}}+\frac{b v(1-\alpha) W_{j j} W^{\frac{1-\alpha}{\beta}}-1}{\beta} \\
& +\frac{b v(1-\alpha)}{\beta} \frac{1-\alpha-\beta}{\beta} W^{\frac{1-\alpha}{\beta}-2} W_{j}^{2}-\frac{\gamma W_{j j}}{W}+\frac{\gamma W_{j}^{2}}{W^{2}}, \\
\frac{\partial G_{0}(x, t)}{\partial t}= & \frac{2 W_{i} W_{i t}}{W^{2}}-\frac{2|\nabla W|^{2} W_{t}}{W^{3}}+b v\left(\frac{1-\alpha}{\beta}\right) W^{\frac{1-\alpha}{\beta}-1} W_{t}-\gamma \frac{W_{t}}{W} .
\end{aligned}
$$


So, we obtain

$$
\begin{aligned}
(\Delta & \left.-\frac{\partial}{\partial t}\right) G_{0} \\
= & \left(\frac{2 W_{i j}^{2}}{W^{2}}-\frac{8 W_{i} W_{j} W_{i j}}{W^{3}}+\frac{6|\nabla W|^{4}}{W^{4}}\right)+\left(\frac{2 W_{i} W_{i j j}}{W^{2}}-\frac{2 W_{i} W_{i t}}{W^{2}}\right) \\
& +\left(-\frac{2|\nabla W|^{2} W_{j j}}{W^{3}}+\frac{2|\nabla W|^{2} W_{t}}{W^{3}}\right)+\left\{\frac{b v(1-\alpha)}{\beta} W^{\frac{1-\alpha}{\beta}}-\gamma\right\} \frac{\left(W_{j j}-W_{t}\right)}{W} \\
& +\frac{|\nabla W|^{2}}{W^{2}}\left\{\frac{b v(1-\alpha)}{\beta} \frac{(1-\alpha-\beta)}{\beta} W^{\frac{1-\alpha}{\beta}}+\gamma\right\} .
\end{aligned}
$$

Using the Ricci identity,

$$
\frac{2 W_{i} W_{i j j}}{W^{2}}=\frac{2 W_{i} W_{j j i}}{W^{2}}+\frac{2 R_{i j} W_{i} W_{j}}{W^{2}}
$$

one then has

$$
\begin{aligned}
\frac{2 W_{i} W_{i j j}}{W^{2}}-\frac{2 W_{i} W_{i t}}{W^{2}} & \\
= & \frac{2\left(\Delta W-W_{t}\right)_{i} W_{i}}{W^{2}}+\frac{2 R_{i j} W_{i} W_{j}}{W^{2}} \\
= & \frac{4(\beta+1)}{\beta} \frac{W_{i} W_{j} W_{i j}}{W^{3}}-\frac{2(\beta+1)}{\beta} \frac{|\nabla W|^{4}}{W^{4}}-\frac{2 a|\nabla W|^{2}}{W^{2}} \ln W \\
& -\frac{2 a|\nabla W|^{2}}{W^{2}}+2 \beta b\left(1+\frac{1-\alpha}{\beta}\right) W^{\frac{1-\alpha}{\beta}} \frac{|\nabla W|^{2}}{W^{2}}+\frac{2 R_{i j} W_{i} W_{j}}{W^{2}} .
\end{aligned}
$$

One also has

$$
\nabla G_{0} \cdot \nabla \ln W=\frac{2 W_{i j} W_{i} W_{j}}{W^{3}}-\frac{2|\nabla W|^{4}}{W^{4}}+\frac{b v(1-\alpha)}{\beta} \frac{|\nabla W|^{2}}{W^{2}} W^{\frac{1-\alpha}{\beta}}-\frac{\gamma|\nabla W|^{2}}{W^{2}}
$$

Multiplying both of the above equality by $2(\beta+1) / \beta$, one then has

$$
\begin{aligned}
& \frac{4(\beta+1)}{\beta} \frac{W_{i j} W_{i} W_{j}}{W^{3}}-\frac{4(\beta+1)}{\beta} \frac{|\nabla W|^{4}}{W^{4}} \\
& =\frac{2(\beta+1)}{\beta} \nabla G_{0} \cdot \nabla \ln W-\frac{2 b v(\beta+1)}{\beta} \frac{(1-\alpha)}{\beta} \frac{|\nabla W|^{2}}{W^{2}} W^{\frac{1-\alpha}{\beta}}+\frac{2 \gamma(\beta+1)}{\beta} \frac{|\nabla W|^{2}}{W^{2}} .
\end{aligned}
$$


Substituting the above equality into (2.6), one obtains

$$
\begin{aligned}
& \frac{2 W_{i} W_{i j j}}{W^{2}}-\frac{2 W_{i} W_{i t}}{W^{2}} \\
& =\frac{2(\beta+1)}{\beta} \nabla G_{0} \cdot \nabla \ln W+\frac{2(\beta+1)}{\beta} \frac{|\nabla W|^{4}}{W^{4}}+\frac{2 \gamma(\beta+1)}{\beta} \frac{|\nabla W|^{2}}{W^{2}} \\
& \quad-\frac{2 b v(\beta+1)}{\beta} \frac{(1-\alpha)}{\beta} \frac{|\nabla W|^{2}}{W^{2}} W^{\frac{1-\alpha}{\beta}}-\frac{2 a|\nabla W|^{2}}{W^{2}} \ln W-\frac{2 a|\nabla W|^{2}}{W^{2}} \\
& \quad+2 \beta b\left(1+\frac{1-\alpha}{\beta}\right) W^{\frac{1-\alpha}{\beta}} \frac{|\nabla W|^{2}}{W^{2}}+\frac{2 R_{i j} W_{i} W_{j}}{W^{2}} .
\end{aligned}
$$

Again,

$$
\begin{aligned}
- & \frac{2|\nabla W|^{2} W_{j j}}{W^{3}}+\frac{2|\nabla W|^{2} W_{t}}{W^{3}} \\
= & -\frac{2|\nabla W|^{2}\left(\Delta W-W_{t}\right)}{W^{3}} \\
= & -\frac{2|\nabla W|^{2}}{W^{3}}\left(\frac{\beta+1}{\beta} \frac{|\nabla W|^{2}}{W}-a W \ln W+\beta b W^{1+\frac{1-\alpha}{\beta}}\right) \\
= & -\frac{2(\beta+1)}{\beta} \frac{|\nabla W|^{4}}{W^{4}}+\frac{2 a|\nabla W|^{2}}{W^{2}} \ln W-\frac{2 \beta b|\nabla W|^{2}}{W^{2}} W^{\frac{1-\alpha}{\beta}} .
\end{aligned}
$$

Finally, since

$$
\frac{2 \varepsilon W_{i j}^{2}}{W^{2}}-\frac{4 W_{i} W_{j} W_{i j}}{W^{3}}+\frac{2 W_{i}^{4}}{\varepsilon W^{4}}=\left(\frac{\sqrt{2 \varepsilon} W_{i j}}{W}-\frac{\sqrt{2} W_{i} W_{j}}{\sqrt{\varepsilon} W^{2}}\right)^{2}>0
$$

and

$$
W_{i j}^{2} \geq \frac{\left(W_{i i}\right)^{2}}{n}
$$

we have

$$
\begin{aligned}
& \frac{2 W_{i j}^{2}}{W^{2}}-\frac{8 W_{i} W_{j} W_{i j}}{W^{3}}+\frac{6|\nabla W|^{4}}{W^{4}} \\
& \geq \frac{2(1-\varepsilon) W_{i j}^{2}}{W^{2}}-\frac{4 W_{i} W_{j} W_{i j}}{W^{3}}+\left(6-\frac{2}{\varepsilon}\right) \frac{W_{i}^{4}}{W^{4}} \\
& \geq \frac{2(1-\varepsilon)}{n}\left(\frac{\Delta W}{W}\right)^{2}-4\left(\frac{W_{i} W_{j} W_{i j}}{W}-\frac{W_{i}^{4}}{W^{4}}\right)-2\left(\frac{1}{\varepsilon}-1\right) \frac{W_{i}^{4}}{W^{4}},
\end{aligned}
$$


where $0<\varepsilon<1$. We also notice

$$
\begin{aligned}
& 4\left(\frac{W_{i} W_{j} W_{i j}}{W^{3}}-\frac{|\nabla W|^{4}}{W^{4}}\right) \\
& =2 \nabla G_{0} \nabla \ln W-\frac{2 b v(1-\alpha)}{\beta} \frac{|\nabla W|^{2}}{W^{2}} W^{\frac{1-\alpha}{\beta}}+2 \gamma \frac{|\nabla W|^{2}}{W^{2}} .
\end{aligned}
$$

Substituting the above equality into (2.9), one has

$$
\begin{aligned}
& \frac{2 W_{i j}^{2}}{W^{2}}-\frac{8 W_{i} W_{j} W_{i j}}{W^{3}}+\frac{6|\nabla W|^{4}}{W^{4}} \\
& \geq \frac{2(1-\varepsilon)}{n}\left(\frac{\Delta W}{W}\right)^{2}-2 \nabla G_{0} \nabla \ln W+\frac{2 b v(1-\alpha)}{\beta} \frac{|\nabla W|^{2}}{W^{2}} W^{\frac{1-\alpha}{\beta}} \\
& \quad-2 \gamma \frac{|\nabla W|^{2}}{W^{2}}-2\left(\frac{1}{\varepsilon}-1\right) \frac{|\nabla W|^{4}}{W^{4}} .
\end{aligned}
$$

Substituting (2.7), (2.8), and (2.10) into (2.5), one then has

$$
\begin{aligned}
& \left(\Delta-\partial_{t}\right) G_{0} \\
& \geq\left\{\frac{2(1-\varepsilon)}{n}\left(\frac{\Delta W}{W}\right)^{2}-2 \nabla G_{0} \nabla \ln W+\frac{2 b v(1-\alpha)}{\beta} \frac{|\nabla W|^{2}}{W^{2}} W^{\frac{1-\alpha}{\beta}}\right. \\
& \left.-2 \gamma \frac{|\nabla W|^{2}}{W^{2}}-2\left(\frac{1}{\varepsilon}-1\right) \frac{|\nabla W|^{4}}{W^{4}}\right\}+\left\{\frac{2(\beta+1)}{\beta} \nabla G_{0} \cdot \nabla \ln W\right. \\
& +\frac{2(\beta+1)}{\beta} \frac{|\nabla W|^{4}}{W^{4}}+\frac{2 \gamma(\beta+1)}{\beta} \frac{|\nabla W|^{2}}{W^{2}}-\frac{2 a|\nabla W|^{2}}{W^{2}} \ln W \\
& -\frac{2 b v(\beta+1)}{\beta} \frac{(1-\alpha)}{\beta} \frac{|\nabla W|^{2}}{W^{2}} W^{\frac{1-\alpha}{\beta}}-\frac{2 a|\nabla W|^{2}}{W^{2}}+\frac{2 R_{i j} W_{i} W_{j}}{W^{2}} \\
& \left.+2 \beta b\left(1+\frac{1-\alpha}{\beta}\right) W^{\frac{1-\alpha}{\beta}} \frac{|\nabla W|^{2}}{W^{2}}\right\}+\left\{-\frac{2(\beta+1)}{\beta} \frac{|\nabla W|^{4}}{W^{4}}+\frac{2 a|\nabla W|^{2}}{W^{2}} \ln W\right. \\
& \left.-\frac{2 \beta b|\nabla W|^{2}}{W^{2}} W^{\frac{1-\alpha}{\beta}}\right\}+\frac{|\nabla W|^{2}}{W^{2}}\left\{\frac{b v(1-\alpha)}{\beta} \frac{(1-\alpha-\beta)}{\beta} W^{\frac{1-\alpha}{\beta}}+\gamma\right\} \\
& +b v(1-\alpha) \frac{\beta+1}{\beta^{2}} \frac{|\nabla W|^{2}}{W^{2}} W^{\frac{1-\alpha}{\beta}}-a b v \frac{1-\alpha}{\beta} W^{\frac{1-\alpha}{\beta}} \ln W \\
& +b^{2} v(1-\alpha) W^{\frac{2(1-\alpha)}{\beta}}-\gamma \frac{\beta+1}{\beta} \frac{|\nabla W|^{2}}{W^{2}}+a \gamma \ln W-\beta b \gamma W^{\frac{1-\alpha}{\beta}} \\
& =\frac{2(1-\varepsilon)}{n}\left(\frac{\Delta W}{W}\right)^{2}-2\left(\frac{1}{\varepsilon}-1\right) \frac{|\nabla W|^{4}}{W^{4}}+\frac{2}{\beta} \nabla G_{0} \nabla \ln W \\
& +\frac{2 R_{i j} W_{i} W_{j}}{W^{2}}+2 b(1-\alpha) \frac{|\nabla W|^{2}}{W^{2}} W^{\frac{1-\alpha}{\beta}}-\frac{b \nu \alpha(1-\alpha)}{\beta^{2}} \frac{|\nabla W|^{2}}{W^{2}} W^{\frac{1-\alpha}{\beta}}
\end{aligned}
$$




$$
\begin{aligned}
& +\left(\frac{\gamma}{\beta}-2 a\right) \frac{|\nabla W|^{2}}{W^{2}}-\frac{a b v(1-\alpha)}{\beta} W^{\frac{1-\alpha}{\beta}} \ln W+b^{2} v(1-\alpha) W^{\frac{2(1-\alpha)}{\beta}} \\
& +a \gamma \ln W-\beta b \gamma W^{\frac{1-\alpha}{\beta}} .
\end{aligned}
$$

Combining (2.11) with (2.4), one has

$$
\begin{aligned}
(\Delta & \left.-\partial_{t}\right) G \\
\geq & \frac{2(1-\varepsilon)}{n}\left(\frac{\Delta W}{W}\right)^{2}-2\left(\frac{1}{\varepsilon}-1\right) \frac{|\nabla W|^{4}}{W^{4}}+\frac{2}{\beta} \nabla G \nabla \ln W \\
& +\frac{2 R_{i j} W_{i} W_{j}}{W^{2}}+2 b(1-\alpha) \frac{|\nabla W|^{2}}{W^{2}} W^{\frac{1-\alpha}{\beta}}-\frac{b v \alpha(1-\alpha)}{\beta^{2}} \frac{|\nabla W|^{2}}{W^{2}} W^{\frac{1-\alpha}{\beta}} \\
& +\left(\frac{\gamma}{\beta}-2 a\right) \frac{|\nabla W|^{2}}{W^{2}}-\frac{a b v(1-\alpha)}{\beta} W^{\frac{1-\alpha}{\beta}} \ln W+b^{2} v(1-\alpha) W^{\frac{2(1-\alpha)}{\beta}} \\
& +a \gamma \ln W-\beta b \gamma W^{\frac{1-\alpha}{\beta}}-\frac{\delta a W_{t}}{W}+\delta b(1-\alpha) W^{\frac{1-\alpha}{\beta}} \frac{W_{t}}{W}
\end{aligned}
$$

By means of (2.3), we rewrite

$$
G-\frac{|\nabla W|^{2}}{W^{2}}=b \nu W^{\frac{1-\alpha}{\beta}}-\gamma \ln W+\delta \frac{W_{t}}{W}
$$

Now, we consider the following two cases: (1) $\delta=\frac{v}{\beta}$ and (2) $\delta=\frac{2 v}{\beta}$; consequently, we will obtain two preliminary lemmas, respectively.

Case I: $\delta=\frac{v}{\beta}$. Setting $\gamma=\frac{a v}{\beta}$, one then has

$$
\frac{\beta}{v}\left(G-\frac{|\nabla W|^{2}}{W^{2}}\right)=\left(\beta b W^{\frac{1-\alpha}{\beta}}-a \ln W+\frac{W_{t}}{W}\right) .
$$

Substituting the above equality into (2.2), one has

$$
\frac{\Delta W}{W}=\frac{\beta+1}{\beta} \frac{|\nabla W|^{2}}{W^{2}}+\frac{\beta}{v}\left(G-\frac{|\nabla W|^{2}}{W^{2}}\right)=\frac{\beta}{v} G+\left(\frac{\beta+1}{\beta}-\frac{\beta}{v}\right) \frac{|\nabla W|^{2}}{W^{2}} .
$$

Substituting this into (2.12), one then has

$$
\begin{aligned}
(\Delta & \left.-\partial_{t}\right) G \\
\geq & \frac{2(1-\varepsilon)}{n}\left(\frac{\beta}{v}\right)^{2} G^{2}+4 \frac{1-\varepsilon}{n}\left(\frac{\beta+1}{\beta}-\frac{\beta}{v}\right) \frac{\beta}{v} G \frac{|\nabla W|^{2}}{W^{2}} \\
& +\left[\frac{2(1-\varepsilon)}{n}\left(\frac{\beta+1}{\beta}-\frac{\beta}{v}\right)^{2}-2\left(\frac{1}{\varepsilon}-1\right)\right] \frac{|\nabla W|^{4}}{W^{4}}+\frac{2}{\beta} \nabla G \nabla \ln W \\
& +\frac{2 R_{i j} W_{i} W_{j}}{W^{2}}+2 b(1-\alpha) \frac{|\nabla W|^{2}}{W^{2}} W^{\frac{1-\alpha}{\beta}}-\frac{b v \alpha(1-\alpha)}{\beta^{2}} \frac{|\nabla W|^{2}}{W^{2}} W^{\frac{1-\alpha}{\beta}}
\end{aligned}
$$




$$
\begin{aligned}
& +\left(\frac{a v}{\beta^{2}}-2 a\right) \frac{|\nabla W|^{2}}{W^{2}}-\frac{a b v(1-\alpha)}{\beta} W^{\frac{1-\alpha}{\beta}} \ln W+b^{2} v(1-\alpha) W^{\frac{2(1-\alpha)}{\beta}} \\
& +\frac{a^{2} v}{\beta} \ln W-a b v W^{\frac{1-\alpha}{\beta}}-\frac{a v}{\beta} \frac{W_{t}}{W}+\frac{b v}{\beta}(1-\alpha) W^{\frac{1-\alpha}{\beta}} \frac{W_{t}}{W} \\
& =\frac{2(1-\varepsilon)}{n}\left(\frac{\beta}{v}\right)^{2} G^{2}+4 \frac{1-\varepsilon}{n}\left(\frac{\beta+1}{\beta}-\frac{\beta}{v}\right) \frac{\beta}{v} G \frac{|\nabla W|^{2}}{W^{2}} \\
& +\left[\frac{2(1-\varepsilon)}{n}\left(\frac{\beta+1}{\beta}-\frac{\beta}{v}\right)^{2}-2\left(\frac{1}{\varepsilon}-1\right)\right] \frac{|\nabla W|^{4}}{W^{4}}+\frac{2}{\beta} \nabla G \nabla \ln W \\
& +\frac{2 R_{i j} W_{i} W_{j}}{W^{2}}+b(1-\alpha) \frac{|\nabla W|^{2}}{W^{2}} W^{\frac{1-\alpha}{\beta}}+b(1-\alpha) \frac{|\nabla W|^{2}}{W^{2}} W^{\frac{1-\alpha}{\beta}} \\
& -\frac{b v \alpha(1-\alpha)}{\beta^{2}} \frac{|\nabla W|^{2}}{W^{2}} W^{\frac{1-\alpha}{\beta}}+\left(\frac{a v}{\beta^{2}}-a\right) \frac{|\nabla W|^{2}}{W^{2}}-a \frac{|\nabla W|^{2}}{W^{2}} \\
& -\frac{a b v(1-\alpha)}{\beta} W^{\frac{1-\alpha}{\beta} \ln W}+b^{2} v(1-\alpha) W^{\frac{2(1-\alpha)}{\beta}}+\frac{a^{2} v}{\beta} \ln W \\
& -a b v W^{\frac{1-\alpha}{\beta}}-\frac{a v}{\beta} \frac{W_{t}}{W}+\frac{b v}{\beta}(1-\alpha) W^{\frac{1-\alpha}{\beta}} \frac{W_{t}}{W} \\
& =\frac{2(1-\varepsilon)}{n}\left(\frac{\beta}{v}\right)^{2} G^{2}+4 \frac{1-\varepsilon}{n}\left(\frac{\beta+1}{\beta}-\frac{\beta}{v}\right) \frac{\beta}{v} G \frac{|\nabla W|^{2}}{W^{2}} \\
& +\left[\frac{2(1-\varepsilon)}{n}\left(\frac{\beta+1}{\beta}-\frac{\beta}{v}\right)^{2}-2\left(\frac{1}{\varepsilon}-1\right)\right] \frac{|\nabla W|^{4}}{W^{4}}+\frac{2}{\beta} \nabla G \nabla \ln W \\
& +\frac{2 R_{i j} W_{i} W_{j}}{W^{2}}+b(1-\alpha) W^{\frac{1-\alpha}{\beta}} G-a G+b(1-\alpha)\left(1-\frac{\nu \alpha}{\beta^{2}}\right) \frac{|\nabla W|^{2}}{W^{2}} W^{\frac{1-\alpha}{\beta}} \\
& +\left(\frac{a v}{\beta^{2}}-a\right) \frac{|\nabla W|^{2}}{W^{2}} \text {. }
\end{aligned}
$$

Thus, we obtain the following lemma.

Lemma 2.1 Let $\delta=\frac{v}{\beta}, \gamma=\frac{a v}{\beta}$, and $W$ and $G$ be as in (2.1) and (2.3), respectively.

Then, one has

$$
\begin{aligned}
\left(\Delta-\partial_{t}\right) G \geq & \frac{2(1-\varepsilon)}{n}\left(\frac{\beta}{v}\right)^{2} G^{2}+4 \frac{1-\varepsilon}{n}\left(\frac{\beta+1}{\beta}-\frac{\beta}{v}\right) \frac{\beta}{v} G \frac{|\nabla W|^{2}}{W^{2}} \\
& +\left[\frac{2(1-\varepsilon)}{n}\left(\frac{\beta+1}{\beta}-\frac{\beta}{v}\right)^{2}-2\left(\frac{1}{\varepsilon}-1\right)\right] \frac{|\nabla W|^{4}}{W^{4}} \\
& +\frac{2}{\beta} \nabla G \nabla \ln W+\frac{2 R_{i j} W_{i} W_{j}}{W^{2}}+b(1-\alpha) W^{\frac{1-\alpha}{\beta}} G-a G \\
& +b(1-\alpha)\left(1-\frac{v \alpha}{\beta^{2}}\right) \frac{|\nabla W|^{2}}{W^{2}} W^{\frac{1-\alpha}{\beta}}+\left(\frac{a v}{\beta^{2}}-a\right) \frac{|\nabla W|^{2}}{W^{2}} .
\end{aligned}
$$


Case II: $\delta=\frac{2 v}{\beta}$. Setting $\gamma=\frac{a v}{\beta}$ and $v=\frac{\beta^{2}}{\alpha}$, and assuming $b \geq 0, \alpha<\frac{n}{n-1}$, and $\ln W \leq 0$ (i.e., $\ln u \geq 0$ ), one then has

$$
G=\frac{|\nabla W|^{2}}{W^{2}}+b \nu W^{\frac{1-\alpha}{\beta}}-\gamma \ln W+\frac{2 v}{\beta} \frac{W_{t}}{W},
$$

and so

$$
\frac{W_{t}}{W}=\frac{\beta}{2 v}\left(G-\frac{|\nabla W|^{2}}{W^{2}}-b v W^{\frac{1-\alpha}{\beta}}+\gamma \ln W\right) .
$$

Plugging the equality above into (2.2), one has

$$
\begin{aligned}
\frac{\Delta W}{W}= & \frac{\beta+1}{\beta} \frac{|\nabla W|^{2}}{W^{2}}-a \ln W+\beta b W^{\frac{1-\alpha}{\beta}}+\frac{W_{t}}{W} \\
= & \frac{\beta+1}{\beta} \frac{|\nabla W|^{2}}{W^{2}}-a \ln W+\beta b W^{\frac{1-\alpha}{\beta}}+\frac{\beta}{2 v} G \\
& -\frac{\beta}{2 v} \frac{|\nabla W|^{2}}{W^{2}}-\frac{\beta b W^{\frac{1-\alpha}{\beta}}}{2}+\frac{\beta \gamma \ln W}{2 v} \\
= & \left(\frac{\beta+1}{\beta}-\frac{\beta}{2 v}\right) \frac{|\nabla W|^{2}}{W^{2}}+\frac{\beta}{2 v} G+\frac{\beta b}{2} W^{\frac{1-\alpha}{\beta}}-\frac{a \ln W}{2},
\end{aligned}
$$

and so

$$
\begin{aligned}
& \left(\frac{\Delta W}{W}\right)^{2}=\left(\frac{\beta+1}{\beta}-\frac{\beta}{2 v}\right)^{2} \frac{|\nabla W|^{4}}{W^{4}}+\frac{\beta^{2} b^{2}}{4} W^{\frac{2(1-\alpha)}{\beta}}+\frac{\beta^{2}}{4 v^{2}} G^{2}+\frac{a^{2}(\ln W)^{2}}{4} \\
& +\left(\frac{\beta+1}{\beta}-\frac{\beta}{2 v}\right) \frac{\beta}{v} \frac{|\nabla W|^{2}}{W^{2}} G+\left(\frac{\beta+1}{\beta}-\frac{\beta}{2 v}\right) \frac{|\nabla W|^{2}}{W^{2}} \beta b W^{\frac{(1-\alpha)}{\beta}} \\
& -\left(\frac{\beta+1}{\beta}-\frac{\beta}{2 v}\right) \frac{|\nabla W|^{2}}{W^{2}} a \ln W+\frac{\beta^{2} b}{2 v} G W^{\frac{1-\alpha}{\beta}} \\
& -\frac{a \beta G \ln W}{2 v}-\frac{a b \beta W^{\frac{(1-\alpha)}{\beta}} \ln W}{2} \\
& \geq\left(\frac{\beta+1}{\beta}-\frac{\beta}{2 v}\right)^{2} \frac{|\nabla W|^{4}}{W^{4}}+\frac{\beta^{2}}{4 v^{2}} G^{2}+\frac{\beta^{2} b}{2 v} G W^{\frac{1-\alpha}{\beta}} \\
& +\left(\frac{\beta+1}{\beta}-\frac{\beta}{2 v}\right) \frac{\beta}{v} \frac{|\nabla W|^{2}}{W^{2}} G+\left(\frac{\beta+1}{\beta}-\frac{\beta}{2 v}\right) \frac{|\nabla W|^{2}}{W^{2}} \beta b W^{\frac{(1-\alpha)}{\beta}} .
\end{aligned}
$$


Substituting the above inequality into (2.12), one has

$$
\begin{aligned}
& \left(\Delta-\frac{\partial}{\partial t}\right) G \\
& \geq \frac{(1-\varepsilon)}{n} \frac{\beta^{2}}{2 v^{2}} G^{2}+\frac{2(1-\varepsilon)}{n}\left(\frac{\beta+1}{\beta}-\frac{\beta}{2 v}\right) \frac{\beta}{v} \frac{|\nabla W|^{2}}{W^{2}} G \\
& +\left[\left(\frac{\beta+1}{\beta}-\frac{\beta}{2 v}\right)^{2} \frac{2(1-\varepsilon)}{n}-2\left(\frac{1}{\epsilon}-1\right)\right] \frac{|\nabla W|^{4}}{W^{4}}+\frac{(1-\varepsilon)}{n v} \beta^{2} b G W^{\frac{1-\alpha}{\beta}} \\
& +\frac{2(1-\varepsilon) \beta b}{n}\left(\frac{\beta+1}{\beta}-\frac{\beta}{2 v}\right) \frac{|\nabla W|^{2}}{W^{2}} W^{\frac{1-\alpha}{\beta}}+\frac{2}{\beta} \nabla G \nabla \ln W \\
& +\frac{2 R_{i j} W_{i} W_{j}}{W^{2}}+2 b(1-\alpha) \frac{|\nabla W|^{2}}{W^{2}} W^{\frac{1-\alpha}{\beta}}-\frac{b v \alpha(1-\alpha)}{\beta^{2}} \frac{|\nabla W|^{2}}{W^{2}} W^{\frac{1-\alpha}{\beta}} \\
& +\left(\frac{\gamma}{\beta}-2 a\right) \frac{|\nabla W|^{2}}{W^{2}}-\frac{a b v(1-\alpha)}{\beta} W^{\frac{1-\alpha}{\beta}} \ln W+b^{2} v(1-\alpha) W^{\frac{2(1-\alpha)}{\beta}} \\
& +a \gamma \ln W-\beta b \gamma W^{\frac{1-\alpha}{\beta}}-\frac{2 v a}{\beta} \frac{W_{t}}{W}+\frac{2 v}{\beta} b(1-\alpha) W^{\frac{1-\alpha}{\beta}} \frac{W_{t}}{W} .
\end{aligned}
$$

Supposing $G\left(x_{0}\right)>0$ for a certain point $x_{0} \in M$, we then have at $x_{0}$

$$
\begin{aligned}
& 2 b(1-\alpha) \frac{|\nabla W|^{2}}{W^{2}} W^{\frac{1-\alpha}{\beta}}-\frac{b v \alpha(1-\alpha)}{\beta^{2}} \frac{|\nabla W|^{2}}{W^{2}} W^{\frac{1-\alpha}{\beta}}-\frac{a b \nu(1-\alpha)}{\beta} W^{\frac{1-\alpha}{\beta}} \ln W \\
& +b^{2} v(1-\alpha) W^{\frac{2(1-\alpha)}{\beta}}+\frac{2 v}{\beta} b(1-\alpha) W^{\frac{1-\alpha}{\beta}} \frac{W_{t}}{W}+\frac{(1-\varepsilon) \alpha}{n} b W^{\frac{1-\alpha}{\beta}} G \\
& =b(1-\alpha) W^{\frac{1-\alpha}{\beta}}\left(\frac{|\nabla W|^{2}}{W^{2}}-\gamma \ln W+v b W^{\frac{1-\alpha}{\beta}}+\frac{2 v}{\beta} \frac{W_{t}}{W}\right)+\frac{(1-\varepsilon) \alpha}{n} b W^{\frac{1-\alpha}{\beta}} G \\
& =b(1-\alpha) W^{\frac{1-\alpha}{\beta}} G+\frac{(1-\varepsilon) \alpha}{n} b W^{\frac{1-\alpha}{\beta}} G \\
& =b W^{\frac{1-\alpha}{\beta}} G\left(1-\alpha+\frac{(1-\varepsilon) \alpha}{n}\right) \geq 0 .
\end{aligned}
$$

We also observe

$$
\left(-a \frac{|\nabla W|^{2}}{W^{2}}+a \gamma \ln W-\gamma b \beta W^{\frac{1-\alpha}{\beta}}-\frac{2 v a}{\beta} \frac{W_{t}}{W}\right)=-a G .
$$

Combining this with (2.14) and (2.15), one has

$$
\left(\Delta-\frac{\partial}{\partial t}\right) G \geq \frac{(1-\varepsilon)}{n} \frac{\alpha^{2}}{2 \beta^{2}} G^{2}+\frac{2(1-\varepsilon) \alpha}{n}\left(\frac{\beta+1}{\beta^{2}}-\frac{\alpha}{2 \beta^{2}}\right) \frac{|\nabla W|^{2}}{W^{2}} G
$$




$$
\begin{aligned}
& +\left[\left(\frac{\beta+1}{\beta}-\frac{\alpha}{2 \beta}\right)^{2} \frac{2(1-\varepsilon)}{n}-2\left(\frac{1}{\varepsilon}-1\right)\right] \frac{|\nabla W|^{4}}{W^{4}} \\
& +\frac{2}{\beta} \nabla G \nabla \ln W+\frac{2 R_{i j} W_{i} W_{j}}{W^{2}}+a\left(\frac{1}{\alpha}-1\right) \frac{|\nabla W|^{2}}{W^{2}}-a G .
\end{aligned}
$$

Then, we have the following lemma.

Lemma 2.2 Let $W$ and $G$ be as in (2.1) and (2.3), respectively, and satisfy $\ln W \leq 0$ and $G\left(x_{0}\right)>0$ for some point $x_{0} \in M$. Set $\delta=\frac{2 v}{\beta}$ and $\gamma=\frac{a v}{\beta}$, and assume $a \geq 0$, $b \geq 0$ and $0<\alpha<\frac{n}{n-1}$. Then, we have at $x_{0}$

$$
\begin{aligned}
\left(\Delta-\frac{\partial}{\partial t}\right) G \geq & \frac{(1-\varepsilon)}{n} \frac{\alpha^{2}}{2 \beta^{2}} G^{2}+\frac{2(1-\varepsilon) \alpha}{n}\left(\frac{\beta+1}{\beta^{2}}-\frac{\alpha}{2 \beta^{2}}\right) \frac{|\nabla W|^{2}}{W^{2}} G \\
& +\left[\left(\frac{\beta+1}{\beta}-\frac{\alpha}{2 \beta}\right)^{2} \frac{2(1-\varepsilon)}{n}-2\left(\frac{1}{\varepsilon}-1\right)\right] \frac{|\nabla W|^{4}}{W^{4}}+\frac{2}{\beta} \nabla G \nabla \ln W \\
& +\frac{2 R_{i j} W_{i} W_{j}}{W^{2}}+a\left(\frac{1}{\alpha}-1\right) \frac{|\nabla W|^{2}}{W^{2}}-a G
\end{aligned}
$$

\section{Gradient Estimates}

Using the lemmas in the previous section and the maximum principle, we can show the gradient estimate in Theorem B.

Let $M$ be an $n$-dimensional complete noncompact Riemannian manifold with Ricci curvature bounded below by $-K(K \geq 0)$. Define a $C^{2}$ cut-off function $\eta=\eta(t)$ on $[0,+\infty)$ as follows:

$$
\eta(t)= \begin{cases}1, & t \in[0,1] \\ >0, & t \in(1,2), \\ 0, & t \in[2,+\infty)\end{cases}
$$

satisfying $\forall t>0,-C \eta^{1 / 2}(t) \leq \eta^{\prime}(t) \leq 0, \eta^{\prime \prime} \geq-C$, here $C$ is some positive constant. We use $\rho(x)$ to denote the geodesic distance of $M$ between a fixed point $p$ and $x$, and set

$$
\psi(x)=\eta\left(\frac{\rho(x)}{R}\right) .
$$

Then, we have

$$
\frac{|\nabla \psi|^{2}}{\psi}=\frac{\left|\eta^{\prime}\right|^{2}}{R^{2} \eta} \leq \frac{C^{2}}{R^{2}} .
$$


By the Laplacian comparison theorem, one has

$$
\Delta \psi(x)=\frac{\eta^{\prime \prime}|\nabla \rho|^{2}}{R^{2}}+\frac{\eta^{\prime} \Delta \rho}{R} \geq \frac{-n C}{R^{2}}-\frac{(n-1) C \sqrt{K}}{R} .
$$

Set $F(x, t)=t G(x, t)$. A standard argument shows (cf. [1] or [2]) that we may assume that the function $\psi(x) F(x, t)$ defined in the geodesic ball $B_{p}(2 R)$ is smooth. Let $\left(x_{0}, t_{0}\right)$ be the maximum point of $\psi(x) F(x, t)$ in $Q_{2 R, T}=B_{p}(2 R) \times[0, T]$. Without loss of generality, we may assume that $\psi\left(x_{0}\right) F\left(x_{0}, t_{0}\right)>0$. By means of the maximum principle, one has at $\left(x_{0}, t_{0}\right)$

$$
\begin{aligned}
& \nabla(\psi F)=0, \\
& \frac{\partial(\psi F)}{\partial t} \geq 0, \\
& \Delta(\psi F) \leq 0 .
\end{aligned}
$$

In the following, unless specified otherwise, all computation and argument are restricted to the point $\left(x_{0}, t_{0}\right)$. By (3.3), one has

$$
\nabla F=-\frac{\nabla \psi}{\psi} F
$$

By (3.5), one has

$$
\Delta \psi \cdot F+2 \nabla \psi \cdot \nabla F+\psi \cdot \Delta F \leq 0
$$

Using (3.4), we obtain

$$
\Delta \psi \cdot F+2 \nabla \psi \cdot \nabla F+\psi\left(\Delta-\frac{\partial}{\partial t}\right) F \leq 0
$$

A direct computation also shows

$$
\left(\Delta-\frac{\partial}{\partial t}\right) F=t \Delta G-G-t G_{t}=t\left(\Delta-\frac{\partial}{\partial t}\right) G-\frac{F}{t} .
$$

Thus, we get

$$
0 \geq \Delta \psi \cdot F-\frac{2|\nabla \psi|^{2}}{\psi} F+t \psi\left(\Delta-\frac{\partial}{\partial t}\right) G-\frac{\psi F}{t}
$$

Similar to the previous section, the following argument will be divided into two cases corresponding to Lemmas 2.1 and 2.2, respectively.

Case I: $\delta=\frac{v}{\beta}$. This will be divided into three cases: (1) $0<\alpha<1$, (2) $\alpha=1$, (3) $1<\alpha<+\infty$. For this case, we set $M_{1}=\sup _{(x, t) \in Q_{2 R, T}} u^{\alpha-1}(x, t)$ in the following argument. 
Case I.1: $0<\alpha<1$. Take $v=\frac{\beta^{2}}{\alpha}$. Then $\frac{a v}{\beta^{2}}-a=\frac{a}{\alpha}-a, 1-\frac{v \alpha}{\beta^{2}}=0, \frac{\beta}{v}=\frac{\alpha}{\beta}$. By (2.13) in Lemma 2.1, one has

$$
\begin{aligned}
(\Delta & \left.-\frac{\partial}{\partial t}\right) F \\
\geq & 2 \frac{1-\varepsilon}{n}\left(\frac{\alpha}{\beta}\right)^{2} \frac{F^{2}}{t}+4 \frac{1-\varepsilon}{n}\left(1+\frac{1-\alpha}{\beta}\right) \frac{\alpha}{\beta} F \frac{|\nabla W|^{2}}{W^{2}}+\frac{2}{\beta} \nabla F \nabla \ln W \\
+ & {\left[\frac{2(1-\varepsilon)}{n}\left(1+\frac{1-\alpha}{\beta}\right)^{2}-2\left(\frac{1}{\varepsilon}-1\right)\right] \frac{|\nabla W|^{4}}{W^{4}} t-2 K t \frac{|\nabla W|^{2}}{W^{2}} } \\
& -|b| M_{1}(1-\alpha) F-|a| F-\frac{F}{t}-|a|\left(\frac{1}{\alpha}-1\right) \frac{|\nabla W|^{2}}{W^{2}} t, \\
= & 2 \frac{1-\varepsilon}{n}\left(\frac{\alpha}{\beta}\right)^{2} \frac{F^{2}}{t}+4 \frac{1-\varepsilon}{n}\left(1+\frac{1-\alpha}{\beta}\right) \frac{\alpha}{\beta} F \frac{|\nabla W|^{2}}{W^{2}}+\frac{2}{\beta} \nabla F \nabla \ln W \\
+ & {\left[\frac{2(1-\varepsilon)}{n}\left(1+\frac{1-\alpha}{\beta}\right)^{2}-2\left(\frac{1}{\varepsilon}-1\right)\right] \frac{|\nabla W|^{4}}{W^{4}} t } \\
& -\left[2 K+|a|\left(\frac{1}{\alpha}-1\right)\right] t \frac{|\nabla W|^{2}}{W^{2}}-|b| M_{1}(1-\alpha) F-|a| F-\frac{F}{t} .
\end{aligned}
$$

Take $\beta>0$ such that $\frac{1}{\beta}>\frac{n}{2 \varepsilon(1-\alpha)}$. Substituting the following inequality

$$
\begin{aligned}
& -\left(2 K+\frac{|a|}{\alpha}-|a|\right) t \frac{|\nabla W|^{2}}{W^{2}} \\
& \geq-\left(K+\frac{|a|}{2 \alpha}-\frac{|a|}{2}\right)^{2} \frac{n \beta^{2} t}{2(1-\varepsilon)(1-\alpha)^{2}}-\frac{2(1-\varepsilon)(1-\alpha)^{2}}{n \beta^{2}} \frac{|\nabla W|^{4}}{W^{4}} t
\end{aligned}
$$

into (3.7) and using (3.3), one then has

$$
\begin{aligned}
(\Delta & \left.-\frac{\partial}{\partial t}\right) F \\
\geq & 2 \frac{1-\varepsilon}{n}\left(\frac{\alpha}{\beta}\right)^{2} \frac{F^{2}}{t}+4 \frac{1-\varepsilon}{n}\left(1+\frac{1-\alpha}{\beta}\right) \frac{\alpha}{\beta} F \frac{|\nabla W|^{2}}{W^{2}}-\frac{2 F}{\beta} \frac{\nabla \psi}{\psi} \frac{\nabla W}{W} \\
& -b M_{1}(1-\alpha) F-a F-\frac{F}{t}-\left(K+\frac{|a|}{2 \alpha}-\frac{|a|}{2}\right)^{2} \frac{n \beta^{2} t}{2(1-\varepsilon)(1-\alpha)^{2}}
\end{aligned}
$$


Plugging the above inequality into (3.6) and using (3.1) and (3.2), we obtain

$$
\begin{aligned}
0 \geq & 2 \frac{1-\varepsilon}{n}\left(\frac{\alpha}{\beta}\right)^{2} \frac{\psi F^{2}}{t}+4 \frac{1-\varepsilon}{n}\left(1+\frac{1-\alpha}{\beta}\right) \frac{\alpha}{\beta} \psi F \frac{|\nabla W|^{2}}{W^{2}}-\frac{2 F}{\beta} \frac{\nabla \psi \nabla W}{W} \\
& -\left(\frac{n c}{R^{2}}+\frac{(n-1) \sqrt{K} C}{R}+\frac{2 C^{2}}{R^{2}}\right) F-|b| M_{1}(1-\alpha) \psi F-|a| \psi F-\frac{\psi F}{t} \\
& -\left(K+\frac{|a|}{2 \alpha}-\frac{|a|}{2}\right)^{2} \frac{n \beta^{2} t \psi}{2(1-\varepsilon)(1-\alpha)^{2}}
\end{aligned}
$$

On the other hand, multiplying (3.8) by $\psi t$ and using the following inequality

$$
\begin{aligned}
& \frac{2 F}{\beta} \frac{\nabla \psi \nabla W}{W} \\
& \quad \leq \frac{4(1-\varepsilon)}{n} \frac{\alpha(1-\alpha)}{\beta^{2}} \psi F \frac{|\nabla W|^{2}}{W^{2}}+\frac{n F}{4(1-\varepsilon)(1-\alpha) \alpha} \frac{|\nabla \psi|^{2}}{\psi} \\
& \quad \leq \frac{4(1-\varepsilon)}{n} \frac{\alpha(1-\alpha)}{\beta^{2}} \psi F \frac{|\nabla W|^{2}}{W^{2}}+\frac{n F}{4(1-\varepsilon)(1-\alpha) \alpha} \frac{C^{2}}{R^{2}}
\end{aligned}
$$

we obtain

$$
\begin{aligned}
0 \geq & 2 \frac{1-\varepsilon}{n}\left(\frac{\alpha}{\beta}\right)^{2} \psi^{2} F^{2}-|b| M_{1}(1-\alpha) \psi t F-|a| \psi t F-\psi F \\
& -\left(\frac{n}{4(1-\varepsilon)(1-\alpha) \alpha} \frac{C^{2}}{R^{2}}+\frac{n c}{R^{2}}+\frac{(n-1) C \sqrt{K}}{R}+\frac{2 C^{2}}{R^{2}}\right) t \psi F \\
& -\left(K+\frac{|a|}{2 \alpha}-\frac{|a|}{2}\right)^{2} \frac{n \beta^{2} t^{2} \psi^{2}}{2(1-\varepsilon)(1-\alpha)^{2}} \\
= & 2 \frac{1-\varepsilon}{n}\left(\frac{\alpha}{\beta}\right)^{2} \psi^{2} F^{2}-\psi F-\left\{\frac{n}{4(1-\varepsilon)(1-\alpha) \alpha} \frac{C^{2}}{R^{2}}+\frac{n c}{R^{2}}\right. \\
& \left.+\frac{(n-1) C \sqrt{K}}{R}+\frac{2 C^{2}}{R^{2}}\right\} t \psi F+\left(|b| M_{1}(1-\alpha)+|a|\right) t \psi F \\
& -\left(K+\frac{|a|}{2 \alpha}-\frac{|a|}{2}\right)^{2} \frac{n \beta^{2} t^{2} \psi^{2}}{2(1-\varepsilon)(1-\alpha)^{2}}
\end{aligned}
$$

which implies

$$
\begin{aligned}
& \frac{|\nabla W|^{2}}{W^{2}}+\frac{\beta^{2} b}{\alpha} W^{\frac{1-\alpha}{\beta}}-\frac{a \beta}{\alpha} \ln W+\frac{\beta}{\alpha} \frac{W_{t}}{W} \\
& =\beta^{2}\left(\frac{|\nabla u|^{2}}{u^{2}}+\frac{b}{\alpha} u^{\alpha-1}+\frac{a}{\alpha} \ln u-\frac{1}{\alpha} \frac{u_{t}}{u}\right) \\
& \leq \frac{n}{2(1-\varepsilon)} \frac{\beta^{2}}{\alpha^{2}} \frac{1}{t}+\left(|b| M_{1}(1-\alpha)+|a|\right) \frac{n}{2(1-\varepsilon)} \frac{\beta^{2}}{\alpha^{2}}
\end{aligned}
$$




$$
\begin{aligned}
& +\left(\frac{n}{4(1-\varepsilon)(1-\alpha) \alpha} \frac{C^{2}}{R^{2}}+\frac{n c}{R^{2}}+\frac{(n-1) C \sqrt{K}}{R}+\frac{2 C^{2}}{R^{2}}\right) \frac{n}{2(1-\varepsilon)} \frac{\beta^{2}}{\alpha^{2}} \\
& +\left(K+\frac{|a|}{2 \alpha}-\frac{|a|}{2}\right) \frac{n \beta^{2}}{2(1-\varepsilon)(1-\alpha) \alpha} .
\end{aligned}
$$

Assuming $\varepsilon$ sufficiently small, we obtain

$$
\begin{aligned}
& \frac{|\nabla u|^{2}}{u^{2}}+\frac{b}{\alpha} u^{\alpha-1}+\frac{a}{\alpha} \ln u-\frac{1}{\alpha} \frac{u_{t}}{u} \\
& \leq \frac{n}{2 t \alpha^{2}}+\left(|b| M_{1}(1-\alpha)+|a|\right) \frac{n}{2 \alpha^{2}}+\left(K+\frac{|a|}{2 \alpha}-\frac{|a|}{2}\right) \frac{n}{2(1-\alpha) \alpha} \\
& \quad+\left(\frac{n}{4(1-\alpha) \alpha} \frac{C^{2}}{R^{2}}+\frac{n c}{R^{2}}+\frac{(n-1) C \sqrt{K}}{R}+\frac{2 C^{2}}{R^{2}}\right) \frac{n}{2 \alpha^{2}} .
\end{aligned}
$$

Case I.2: $\alpha=1$. Set $v=2 \beta^{2}, \delta=\frac{v}{\beta}=2 \beta$, and $\gamma=\frac{a v}{\beta}=2 a \beta$. By (2.13), we obtain

$$
\begin{aligned}
\left(\Delta-\frac{\partial}{\partial t}\right) G \geq & \frac{2(1-\varepsilon)}{n}\left(\frac{1}{2 \beta}\right)^{2} G^{2}+4 \frac{1-\varepsilon}{n}\left(1+\frac{1}{2 \beta}\right) \frac{1}{2 \beta} G \frac{|\nabla W|^{2}}{W^{2}} \\
& +\left[\frac{2(1-\varepsilon)}{n}\left(1+\frac{1}{2 \beta}\right)^{2}-2\left(\frac{1}{\varepsilon}-1\right)\right] \frac{|\nabla W|^{4}}{W^{4}} \\
& +\frac{2}{\beta} \nabla G \nabla \ln W-2 K \frac{|\nabla W|^{2}}{W^{2}}-|a| G-|a| \frac{|\nabla W|^{2}}{W^{2}},
\end{aligned}
$$

one then has

$$
\begin{aligned}
\left(\Delta-\frac{\partial}{\partial t}\right) F \geq & \frac{2(1-\varepsilon)}{n}\left(\frac{1}{2 \beta}\right)^{2} \frac{F^{2}}{t}+2 \frac{1-\varepsilon}{n}\left(1+\frac{1}{2 \beta}\right) \frac{1}{\beta} F \frac{|\nabla W|^{2}}{W^{2}} \\
+ & {\left[\frac{2(1-\varepsilon)}{n}\left(1+\frac{1}{2 \beta}\right)^{2}-2\left(\frac{1}{\varepsilon}-1\right)\right] \frac{|\nabla W|^{4}}{W^{4}} t } \\
& -\frac{2 F}{\beta} \frac{\nabla \psi \nabla W}{\psi W}-(2 K+|a|) t \frac{|\nabla W|^{2}}{W^{2}}-|a| F-\frac{F}{t} .
\end{aligned}
$$

Take $\beta>0$ such that $\frac{1}{\beta}>\frac{n}{\varepsilon}$. Substituting (3.10) into (3.6) and using the following inequality

$$
(2 K+|a|) t \frac{|\nabla W|^{2}}{W^{2}} \leq \frac{1-\varepsilon}{n} \frac{1}{2 \beta^{2}} \frac{|\nabla W|^{4}}{W^{4}} t+\frac{2 n \beta^{2}}{1-\varepsilon}\left(K+\frac{|a|}{2}\right)^{2} t
$$


one then has

$$
\begin{aligned}
0 \geq & \frac{(1-\varepsilon)}{2 n \beta^{2}} \frac{\psi F^{2}}{t}+2 \frac{1-\varepsilon}{n}\left(1+\frac{1}{2 \beta}\right) \frac{1}{\beta} \psi F \frac{|\nabla W|^{2}}{W^{2}} \\
& -\left(\frac{n c}{R^{2}}+\frac{(n-1) C \sqrt{K}}{R}+\frac{2 C^{2}}{R^{2}}\right) \psi F-\frac{2 F}{\beta} \frac{\nabla \psi \nabla W}{W} \\
& -|a| \psi F-\frac{\psi F}{t}-\frac{2 n \beta^{2}}{1-\varepsilon}\left(K+\frac{|a|}{2}\right)^{2} t \psi
\end{aligned}
$$

Substituting (3.9) into (3.11) and then multiplying both sides by $\psi t$, we obtain

$$
\begin{aligned}
0 \geq & \frac{(1-\varepsilon)}{n}\left(\frac{1}{2 \beta^{2}}\right) \psi^{2} F^{2}-\left\{\frac{n}{(1-\varepsilon)} \frac{C^{2}}{R^{2}}+\frac{n c}{R^{2}}+\frac{(n-1) C \sqrt{K}}{R}\right. \\
& \left.+\frac{2 C^{2}}{R^{2}}\right\} t \psi F-|a| \psi t F-\psi F-\frac{2 n \beta^{2}}{1-\varepsilon}\left(K+\frac{|a|}{2}\right)^{2} t^{2} \psi^{2} .
\end{aligned}
$$

The above inequality implies that

$$
\begin{aligned}
& \frac{|\nabla W|^{2}}{W^{2}}+2 \beta^{2} b W^{\frac{1-\alpha}{\beta}}-2 a \beta \ln W+2 \beta \frac{W_{t}}{W} \\
& =\beta^{2}\left(\frac{|\nabla u|^{2}}{u^{2}}+2 b u^{\alpha-1}+2 a \ln u-2 \frac{u_{t}}{u}\right) \\
& \leq \frac{2 n \beta^{2}}{(1-\varepsilon)} \frac{1}{t}+|a| \frac{2 n \beta^{2}}{(1-\varepsilon)}+\left(K+\frac{|a|}{2}\right) \frac{2 n \beta^{2}}{(1-\varepsilon)} \\
& \quad+\left(\frac{n}{(1-\varepsilon)} \frac{C^{2}}{R^{2}}+\frac{n c}{R^{2}}+\frac{(n-1) \sqrt{K} C}{R}+\frac{2 C^{2}}{R^{2}}\right) \frac{2 n \beta^{2}}{(1-\varepsilon)} .
\end{aligned}
$$

Taking $\varepsilon$ sufficiently small, we get

$$
\begin{aligned}
& \frac{|\nabla u|^{2}}{u^{2}}+2 b u^{\alpha-1}+2 a \ln u-2 \frac{u_{t}}{u} \\
& \quad \leq \frac{2 n}{t}+2 n|a|+2 n\left(K+\frac{|a|}{2}\right)+2 n\left(n \frac{C^{2}}{R^{2}}+\frac{n c}{R^{2}}+\frac{(n-1) \sqrt{K} C}{R}+\frac{2 C^{2}}{R^{2}}\right) .
\end{aligned}
$$

Case I.3: $1<\alpha<\infty$. Set $v=2 \beta^{2}$. Then $\delta=\frac{v}{\beta}=2 \beta, \gamma=\frac{a v}{\beta}=2 a \beta, \frac{\beta}{v}=\frac{1}{2 \beta}$, $\frac{\beta+1}{\beta}-\frac{\beta}{v}=1+\frac{1}{2 \beta}, b-\frac{b v \alpha}{\beta^{2}}=b(1-2 \alpha)<0$, and $\frac{a v}{\beta^{2}}-a=a$. 
By (2.13), we have

$$
\begin{aligned}
\left(\Delta-\frac{\partial}{\partial t}\right) G \geq & \frac{(1-\varepsilon)}{2 n \beta^{2}} G^{2}+2 \frac{1-\varepsilon}{n}\left(1+\frac{1}{2 \beta}\right) \frac{1}{\beta} G \frac{|\nabla W|^{2}}{W^{2}} \\
& +\left[\frac{2(1-\varepsilon)}{n}\left(1+\frac{1}{2 \beta}\right)^{2}-2\left(\frac{1}{\varepsilon}-1\right)\right] \frac{|\nabla W|^{4}}{W^{4}} \\
& +\frac{2}{\beta} \nabla G \nabla \ln W-2 K \frac{|\nabla W|^{2}}{W^{2}}-|b| M_{1}(\alpha-1) G-|a| G \\
& -|a| \frac{|\nabla W|^{2}}{W^{2}}-|b|(1-\alpha)(1-2 \alpha) M_{1} \frac{|\nabla W|^{2}}{W^{2}},
\end{aligned}
$$

hence,

$$
\begin{aligned}
\left(\Delta-\frac{\partial}{\partial t}\right) F \geq & \frac{(1-\varepsilon)}{2 n \beta^{2}} \frac{F^{2}}{t}+2 \frac{1-\varepsilon}{n}\left(1+\frac{1}{2 \beta}\right) \frac{1}{\beta} F \frac{|\nabla W|^{2}}{W^{2}} \\
& +\left[\frac{2(1-\varepsilon)}{n}\left(1+\frac{1}{2 \beta}\right)^{2}-2\left(\frac{1}{\varepsilon}-1\right)\right] \frac{|\nabla W|^{4}}{W^{4}} t \\
& -\frac{2 F}{\beta} \frac{\nabla \psi}{\psi} \frac{\nabla W}{W}-\frac{F}{t}-|a| F-|b| M_{1}(\alpha-1) F \\
& -\left(2 K+|a|+|b|(1-\alpha)(1-2 \alpha) M_{1}\right) t \frac{|\nabla W|^{2}}{W^{2}} .
\end{aligned}
$$

In the above inequality, we used the following inequality

$$
\begin{aligned}
& \left\{2 K+|a|+|b|(1-\alpha)(1-2 \alpha) M_{1}\right\} t \frac{|\nabla W|^{2}}{W^{2}} \\
& \quad \leq\left(K+\frac{|a|}{2}+\frac{|b|}{2}(1-\alpha)(1-2 \alpha) M_{1}\right)^{2} \frac{2 n \beta^{2}}{(1-\varepsilon)} t+\frac{(1-\varepsilon)}{n} \frac{1}{2 \beta^{2}} \frac{|\nabla W|^{4}}{W^{4}} t
\end{aligned}
$$

Take $\beta>0$ such that $\frac{1}{\beta}>\frac{n}{\varepsilon}$. Substituting (3.12) into (3.6), one then has

$$
\begin{aligned}
0 \geq & \frac{(1-\varepsilon)}{2 n \beta^{2}} \frac{\psi F^{2}}{t}+2 \frac{1-\varepsilon}{n}\left(1+\frac{1}{2 \beta}\right) \frac{1}{\beta} \psi F \frac{|\nabla W|^{2}}{W^{2}} \\
& -\frac{2 F}{\beta} \nabla \psi \frac{\nabla W}{W}-\frac{\psi F}{t}-|a| \psi F-\left(\frac{n c}{R^{2}}+\frac{(n-1) \sqrt{K}}{R}+\frac{2 C^{2}}{R^{2}}\right) t \psi F \\
& -\left(K+\frac{|a|}{2}+\frac{|b|}{2}(1-\alpha)(1-2 \alpha) M_{1}\right)^{2} t \psi \frac{2 n \beta^{2}}{(1-\varepsilon)}-|b| M_{1}(\alpha-1) \psi F .
\end{aligned}
$$


Using (3.9) and multiplying both sides of (3.13) by $\psi t$, one has

$$
\begin{aligned}
0 \geq & \frac{(1-\varepsilon)}{2 n} \psi^{2} F^{2}-\frac{n}{(1-\varepsilon)} t \psi F \frac{C^{2}}{R^{2}} \\
& -\psi F-|a| t \psi F-\left(\frac{n c}{R^{2}}+\frac{(n-1) \sqrt{K}}{R}+\frac{2 C^{2}}{R^{2}}\right) t \psi F-|b|(\alpha-1) M_{1} t \psi F \\
& -\left(K+\frac{|a|}{2}+\frac{|b|}{2}(1-\alpha)(1-2 \alpha) M_{1}\right)^{2} t^{2} \psi^{2} \frac{2 n \beta^{2}}{(1-\varepsilon)}
\end{aligned}
$$

The above inequality implies

$$
\begin{aligned}
& \frac{|\nabla W|^{2}}{W^{2}}+2 \beta^{2} b W^{\frac{1-\alpha}{\beta}}-2 a \beta \ln W+2 \beta \frac{W_{t}}{W} \\
& =\beta^{2}\left(\frac{|\nabla u|^{2}}{u^{2}}+2 b u^{\alpha-1}+2 a \ln u-2 \frac{u_{t}}{u}\right) \\
& \leq \frac{2 n \beta^{2}}{1-\varepsilon} \frac{1}{t}+\frac{2 n \beta^{2}}{1-\varepsilon}\left\{|b| M_{1}(\alpha-1)+|a|\right\} \\
& \quad+\frac{2 n \beta^{2}}{1-\varepsilon}\left\{\frac{n c}{R^{2}}+\frac{(n-1) \sqrt{K}}{R}+\frac{2 C^{2}}{R^{2}}+\frac{n}{(1-\varepsilon)} \frac{C^{2}}{R^{2}}\right\} \\
& \quad+\left(K+\frac{|a|}{2}+\frac{|b|}{2}(1-\alpha)(1-2 \alpha) M_{1}\right) \frac{2 n \beta^{2}}{1-\varepsilon} .
\end{aligned}
$$

Taking $\varepsilon$ sufficiently small, we obtain

$$
\begin{aligned}
& \frac{|\nabla u|^{2}}{u^{2}}+2 b u^{\alpha-1}+2 a \ln u-2 \frac{u_{t}}{u} \\
& \leq \frac{2 n}{t}+2 n\left\{|b| M_{1}(\alpha-1)+|a|\right\} \\
& \quad+2 n\left\{\frac{n c}{R^{2}}+\frac{(n-1) \sqrt{K}}{R}+\frac{2 C^{2}}{R^{2}}+\frac{n C^{2}}{R^{2}}\right\} \\
& \quad+\left(K+\frac{|a|}{2}+\frac{|b|}{2}(1-\alpha)(1-2 \alpha) M_{1}\right) 2 n
\end{aligned}
$$

Summing up the above all, we then get the following local gradient estimate.

Theorem 3.1 Let $M$ be an n-dimensional complete noncompact Riemannian manifold with Ricci curvature bounded below by $-K(K \geq 0)$. Assume $p \in M$ and $B_{p}(2 R)$, the geodesic ball of radius $2 R$ around $p, Q_{2 R, T} \triangleq q B_{p}(2 R) \times[0, T]$. If $u(x, t)$ is a positive solution of (1.6) on $M \times[0, T]$, then for $(x, t) \in Q_{2 R, T}, u(x, t)$ has the following gradient estimates for some positive constant $C>0$. 
(I) If $0<\alpha<1$,

$$
\begin{aligned}
& \frac{|\nabla u|^{2}}{u^{2}}+\frac{b}{\alpha} u^{\alpha-1}+\frac{a}{\alpha} \ln u-\frac{1}{\alpha} \frac{u_{t}}{u} \\
& \leq \frac{n}{2 t \alpha^{2}}+\left(|b| M_{1}(1-\alpha)+|a|\right) \frac{n}{2 \alpha^{2}}+\left(K+\frac{|a|}{2 \alpha}-\frac{|a|}{2}\right) \frac{n}{2(1-\alpha) \alpha} \\
& \quad+\left(\frac{n}{4(1-\alpha) \alpha} \frac{C^{2}}{R^{2}}+\frac{n c}{R^{2}}+\frac{(n-1) C \sqrt{K}}{R}+\frac{2 C^{2}}{R^{2}}\right) \frac{n}{2 \alpha^{2}}
\end{aligned}
$$

(II) If $\alpha=1$,

$$
\begin{aligned}
& \left(\frac{|\nabla u|^{2}}{u^{2}}+2 b u^{\alpha-1}+2 a \ln u-2 \frac{u_{t}}{u}\right) \\
& \leq \frac{2 n}{t}+2 n|a|+2 n\left(K+\frac{|a|}{2}\right) \\
& +2 n\left(n \frac{C^{2}}{R^{2}}+\frac{n c}{R^{2}}+\frac{(n-1) \sqrt{K} C}{R}+\frac{2 C^{2}}{R^{2}}\right) .
\end{aligned}
$$

(III) If $1<\alpha<+\infty$,

$$
\begin{aligned}
& \frac{|\nabla u|^{2}}{u^{2}}+2 b u^{\alpha-1}+2 a \ln u-2 \frac{u_{t}}{u} \\
& \leq \frac{2 n}{t}+2 n\left\{|b| M_{1}(\alpha-1)+|a|\right\} \\
& \quad+2 n\left\{\frac{n c}{R^{2}}+\frac{(n-1) \sqrt{K}}{R}+\frac{2 C^{2}}{R^{2}}+\frac{n C^{2}}{R^{2}}\right\} \\
& \quad+\left(K+\frac{|a|}{2}+\frac{|b|}{2}(1-\alpha)(1-2 \alpha) M_{1}\right) 2 n .
\end{aligned}
$$

If $a \geq 0$ and $b \geq 0$ in (1.6), and letting $R \rightarrow+\infty$, we get

Corollary 3.2 Let $M$ be an n-dimensional complete noncompact Riemannian manifold with Ricci curvature bounded below by $-K(K \geq 0)$. Assume $a \geq 0$ and $b \geq 0$. If $u(x, t)$ is a positive solution of (1.6) on $M \times[0, T]$, then we have the following gradient estimates for some constant $C>0$.

(I) If $0<\alpha<1$,

$$
\begin{aligned}
& \frac{|\nabla u|^{2}}{u^{2}}+\frac{b}{\alpha} u^{\alpha-1}+\frac{a}{\alpha} \ln u-\frac{1}{\alpha} \frac{u_{t}}{u} \\
& \leq \frac{n}{2 t \alpha^{2}}+a \frac{n}{2 \alpha^{2}}+\left(K+\frac{|a|}{2 \alpha}-\frac{|a|}{2}\right) \frac{n}{2(1-\alpha) \alpha}
\end{aligned}
$$


(II) If $1 \leq \alpha<+\infty$,

$$
\begin{aligned}
& \left(\frac{|\nabla u|^{2}}{u^{2}}+2 b u^{\alpha-1}+2 a \ln u-2 \frac{u_{t}}{u}\right) \\
& \leq \frac{2 n}{t}+2 n|a|+2 n\left(K+\frac{|a|}{2}\right) .
\end{aligned}
$$

Case II: $\delta=\frac{2 v}{\beta}$. If $0<\alpha<1$ or $1<\alpha \leq \frac{n}{n-1}$, by (2.16), we have

$$
\begin{aligned}
(\Delta & \left.-\frac{\partial}{\partial t}\right) F=t\left(\Delta-\frac{\partial}{\partial t}\right) G-\frac{F}{t} \\
\geq & \frac{(1-\varepsilon)}{n} \frac{\alpha^{2}}{2 \beta^{2}} G^{2} t+\frac{2(1-\varepsilon) \alpha}{n}\left(\frac{\beta+1}{\beta^{2}}-\frac{\alpha}{2 \beta^{2}}\right) \frac{|\nabla W|^{2}}{W^{2}} G t \\
& +\left[\left(\frac{\beta+1}{\beta}-\frac{\alpha}{2 \beta}\right)^{2} \frac{2(1-\varepsilon)}{n}-2\left(\frac{1}{\varepsilon}-1\right)\right] \frac{|\nabla W|^{4}}{W^{4}} t+\frac{2 t}{\beta} \nabla G \nabla \ln W \\
& +\frac{2 R_{i j} W_{i} W_{j}}{W^{2}} t+a\left(\frac{1}{\alpha}-1\right) \frac{|\nabla W|^{2}}{W^{2}} t-a G t-\frac{F}{t} . \\
\geq & \frac{(1-\varepsilon)}{n} \frac{\alpha^{2}}{2 \beta^{2}} \frac{F^{2}}{t}+\frac{2(1-\varepsilon) \alpha}{n}\left(\frac{\beta+1}{\beta^{2}}-\frac{\alpha}{2 \beta^{2}}\right) \frac{|\nabla W|^{2}}{W^{2}} F \\
& +\left[\left(\frac{\beta+1}{\beta}-\frac{\alpha}{2 \beta}\right)^{2} \frac{2(1-\varepsilon)}{n}-2\left(\frac{1}{\varepsilon}-1\right)\right] \frac{|\nabla W|^{4}}{W^{4}} t+\frac{2}{\beta} \nabla F \nabla \ln W \\
& -2 K \frac{|\nabla W|^{2}}{W^{2}} t-a\left|\frac{1}{\alpha}-1\right| \frac{|\nabla W|^{2}}{W^{2}} t-a F-\frac{F}{t} .
\end{aligned}
$$

Take $\beta>0$ such that $\frac{1}{\beta}>\frac{n}{\varepsilon(2-\alpha)}$. Substituting the following inequality

$$
\begin{aligned}
& -\left(2 K+a\left|\frac{1}{\alpha}-1\right|\right) t \frac{|\nabla W|^{2}}{W^{2}} \\
& \geq-\left(K+\frac{a}{2}\left|\frac{1}{\alpha}-1\right|\right)^{2} \frac{n}{2(1-\varepsilon)}\left(\frac{2 \beta}{2-\alpha}\right)^{2} t-\frac{2(1-\varepsilon)}{n}\left(\frac{2-\alpha}{2 \beta}\right)^{2} \frac{|\nabla W|^{4}}{W^{4}} t
\end{aligned}
$$

into (3.19) and using (3.3), one then has

$$
\begin{aligned}
\left(\Delta-\frac{\partial}{\partial t}\right) F \geq & \frac{(1-\varepsilon)}{n} \frac{\alpha^{2}}{2 \beta^{2}} \frac{F^{2}}{t}+\frac{2(1-\varepsilon) \alpha}{n \beta}\left(1+\frac{1}{\beta}-\frac{\alpha}{2 \beta}\right) \frac{|\nabla W|^{2}}{W^{2}} F \\
& +\frac{2}{\beta} \nabla F \nabla \ln W-a F-\frac{F}{t}-\left(K+\frac{a}{2}\left|\frac{1}{\alpha}-1\right|\right)^{2} \frac{2 n \beta^{2}}{(1-\varepsilon)(2-\alpha)^{2}} t
\end{aligned}
$$


Plugging the above inequality into (3.6), and multiplying both sides by $\psi t$, one obtains

$$
\begin{aligned}
& 0 \geq \frac{(1-\varepsilon)}{n} \frac{\alpha^{2}}{2 \beta^{2}} F^{2} \psi^{2}+\frac{2(1-\varepsilon) \alpha}{n \beta}\left(1+\frac{1}{\beta}-\frac{\alpha}{2 \beta}\right) \frac{|\nabla W|^{2}}{W^{2}} F \psi t \\
& -\left(\frac{n c}{R^{2}}+\frac{(n-1) C \sqrt{K}}{R}+2 \frac{C^{2}}{R^{2}}\right) F \psi t+\frac{2}{\beta} t \psi \nabla F \nabla \ln W \\
& -a \psi F t-\psi F-\left(K+\frac{|a|}{2}\left|\frac{1}{\alpha}-1\right|\right)^{2} \frac{2 n \beta^{2}}{(1-\varepsilon)(2-\alpha)^{2}} t^{2} \\
& =\frac{(1-\varepsilon)}{n} \frac{\alpha^{2}}{2 \beta^{2}} F^{2} \psi^{2}+\frac{2(1-\varepsilon) \alpha}{n \beta}\left(1+\frac{1}{\beta}-\frac{\alpha}{2 \beta}\right) \frac{|\nabla W|^{2}}{W^{2}} F \psi t \\
& -\left(\frac{n c}{R^{2}}+\frac{(n-1) C \sqrt{K}}{R}+2 \frac{C^{2}}{R^{2}}\right) F \psi t-\frac{2}{\beta} t F \nabla \psi \nabla \ln W \\
& -a \psi F t-\psi F-\left(K+\frac{a}{2}\left|\frac{1}{\alpha}-1\right|\right)^{2} \frac{2 n \beta^{2}}{(1-\varepsilon)(2-\alpha)} t^{2} \\
& \geq \frac{(1-\varepsilon)}{n} \frac{\alpha^{2}}{2 \beta^{2}} F^{2} \psi^{2}-\frac{n}{(1-\varepsilon)(2-\alpha) \alpha} \frac{C^{2}}{R^{2}} F \psi t \\
& -\left(\frac{n c}{R^{2}}+\frac{(n-1) C \sqrt{K}}{R}+2 \frac{C^{2}}{R^{2}}\right) F \psi t \\
& -a \psi F t-\psi F-\left(K+\frac{a}{2}\left|\frac{1}{\alpha}-1\right|\right)^{2} \frac{2 n \beta^{2}}{(1-\varepsilon)(2-\alpha)^{2}} t^{2} \\
& =\frac{(1-\varepsilon)}{n} \frac{\alpha^{2}}{2 \beta^{2}} F^{2} \psi^{2}-a \psi F t-\psi F \\
& -\left(\frac{n c}{R^{2}}+\frac{(n-1) C \sqrt{K}}{R}+2 \frac{C^{2}}{R^{2}}+\frac{n}{(1-\varepsilon)(2-\alpha) \alpha} \frac{C^{2}}{R^{2}}\right) F \psi t \\
& -\left(K+\frac{a}{2}\left|\frac{1}{\alpha}-1\right|\right)^{2} \frac{2 n \beta^{2}}{(1-\varepsilon)(2-\alpha)^{2}} t^{2} \text {. }
\end{aligned}
$$

Notice that here, we used the following inequality

$$
-\frac{2 F}{\beta} \frac{\nabla \psi \nabla W}{W} \geq-F \frac{|\nabla \psi|^{2}}{\psi} \frac{n}{(1-\varepsilon)(2-\alpha) \alpha}-\psi F \frac{|\nabla W|^{2}}{W^{2}} \frac{(1-\varepsilon)(2-\alpha) \alpha}{n \beta^{2}}
$$

Then, (3.20) implies

$$
\begin{aligned}
& \frac{|\nabla W|^{2}}{W^{2}}+\frac{b \beta^{2}}{\alpha} W^{\frac{1-\beta}{\alpha}}-\frac{a \beta}{\alpha} \ln W+\delta \frac{W_{t}}{W} \\
& =\beta^{2}\left(\frac{|\nabla u|^{2}}{u^{2}}+\frac{b}{\alpha} u^{\alpha-1}+\frac{a}{\alpha} \ln u-\frac{2}{\alpha} \frac{u_{t}}{u}\right)
\end{aligned}
$$




$$
\begin{aligned}
\leq & \frac{1}{t} \frac{2 n \beta^{2}}{(1-\varepsilon) \alpha^{2}}+\frac{2 n \beta^{2}}{(1-\varepsilon) \alpha^{2}}\left(\frac{n c}{R^{2}}+\frac{(n-1) C \sqrt{K}}{R}+\frac{2 C^{2}}{R^{2}}+a\right. \\
& \left.+\frac{n}{(1-\varepsilon)(2-\alpha) \alpha} \frac{C^{2}}{R^{2}}\right)+\left(K+\frac{a}{2}\left|\frac{1}{\alpha}-1\right|\right) \frac{2 n \beta^{2}}{(2-\alpha)(1-\varepsilon) \alpha} .
\end{aligned}
$$

Letting $\varepsilon$ sufficiently small, we obtain

$$
\begin{aligned}
& \frac{|\nabla u|^{2}}{u^{2}}+\frac{b}{\alpha} u^{\alpha-1}+\frac{a}{\alpha} \ln u-\frac{2}{\alpha} \frac{u_{t}}{u} \\
& \leq \frac{1}{t} \frac{2 n}{\alpha^{2}}+\frac{2 n}{\alpha^{2}}\left(\frac{n c}{R^{2}}+\frac{(n-1) C \sqrt{K}}{R}+\frac{2 C^{2}}{R^{2}}+a\right. \\
& \left.\quad+\frac{2 C^{2}}{R^{2}}+\frac{n}{(2-\alpha) \alpha} \frac{C^{2}}{R^{2}}\right)+\left(K+\frac{a}{2}\left|\frac{1}{\alpha}-1\right|\right) \frac{2 n}{(2-\alpha) \alpha} .
\end{aligned}
$$

If $\alpha=1$, by (2.16), we have

$$
\begin{aligned}
(\Delta & \left.-\frac{\partial}{\partial t}\right) F=t\left(\Delta-\frac{\partial}{\partial t}\right) G-\frac{F}{t} \\
\geq & \frac{(1-\varepsilon)}{2 n \beta^{2}} G^{2} t+\frac{2(1-\varepsilon)}{n}\left(\frac{\beta+1}{\beta^{2}}-\frac{1}{2 \beta^{2}}\right) \frac{|\nabla W|^{2}}{W^{2}} G t \\
& +\left[\left(\frac{\beta+1}{\beta}-\frac{1}{2 \beta}\right)^{2} \frac{2(1-\varepsilon)}{n}-2\left(\frac{1}{\varepsilon}-1\right)\right] \frac{|\nabla W|^{4}}{W^{4}} t+\frac{2 t}{\beta} \nabla G \nabla \ln W \\
& +\frac{2 R_{i j} W_{i} W_{j}}{W^{2}} t-a G t-\frac{F}{t} . \\
\geq & \frac{(1-\varepsilon)}{2 n \beta^{2}} \frac{F^{2}}{t}+\frac{2(1-\varepsilon)}{n}\left(\frac{\beta+1}{\beta^{2}}-\frac{1}{2 \beta^{2}}\right) \frac{|\nabla W|^{2}}{W^{2}} F \\
& +\left[\left(1+\frac{1}{2 \beta}\right)^{2} \frac{2(1-\varepsilon)}{n}-2\left(\frac{1}{\varepsilon}-1\right)\right] \frac{|\nabla W|^{4}}{W^{4}} t+\frac{2}{\beta} \nabla F \nabla \ln W \\
& -2 K \frac{|\nabla W|^{2}}{W^{2}} t-a F-\frac{F}{t} .
\end{aligned}
$$

Take $\beta>0$ such that $\frac{1}{\beta}>\frac{n}{\varepsilon}$. Subsituting the following inequality

$$
-2 K t \frac{|\nabla W|^{2}}{W^{2}} \geq-K^{2} \frac{2 n \beta^{2}}{1-\varepsilon} t-\frac{(1-\varepsilon)}{n} \frac{1}{2 \beta^{2}} \frac{|\nabla W|^{4}}{W^{4}} t
$$


into (3.21) and using (3.3), we then have

$$
\begin{aligned}
\left(\Delta-\frac{\partial}{\partial t}\right) F \geq & \frac{(1-\varepsilon)}{2 n \beta^{2}} \frac{F^{2}}{t}+\frac{2(1-\varepsilon)}{n \beta}\left(1+\frac{1}{2 \beta}\right) \frac{|\nabla W|^{2}}{W^{2}} F \\
& +\frac{2}{\beta} \nabla F \nabla \ln W-a F-\frac{F}{t}-K^{2} \frac{2 n \beta^{2}}{1-\varepsilon} t .
\end{aligned}
$$

Plugging the above inequality into (3.6), and multiplying both sides by $\psi t$, one obtains

$$
\begin{aligned}
& 0 \geq \frac{(1-\varepsilon)}{2 n \beta^{2}} F^{2} \psi^{2}+\frac{2(1-\varepsilon)}{n \beta}\left(1+\frac{1}{2 \beta}\right) \frac{|\nabla W|^{2}}{W^{2}} F \psi t \\
& -\left(\frac{n c}{R^{2}}+\frac{(n-1) C \sqrt{K}}{R}+2 \frac{C^{2}}{R^{2}}\right) F \psi t+\frac{2}{\beta} t \psi \nabla F \nabla \ln W-a \psi F t-\psi F \\
& -K^{2} \frac{2 n \beta^{2}}{1-\varepsilon} t \\
& =\frac{(1-\varepsilon)}{2 n \beta^{2}} F^{2} \psi^{2}+\frac{2(1-\varepsilon)}{n \beta}\left(1+\frac{1}{2 \beta}\right) \frac{|\nabla W|^{2}}{W^{2}} F \psi t \\
& -\left(\frac{n c}{R^{2}}+\frac{(n-1) C \sqrt{K}}{R}+2 \frac{C^{2}}{R^{2}}\right) F \psi t-\frac{2}{\beta} t F \nabla \psi \nabla \ln W-a \psi F t-\psi F \\
& -K^{2} \frac{2 n \beta^{2}}{1-\varepsilon} t \\
& \geq \frac{(1-\varepsilon)}{2 n \beta^{2}} F^{2} \psi^{2}-\frac{n}{(1-\varepsilon)} \frac{C^{2}}{R^{2}} F \psi t \\
& -\left(\frac{n c}{R^{2}}+\frac{(n-1) C \sqrt{K}}{R}+2 \frac{C^{2}}{R^{2}}\right) F \psi t-a \psi F t-\psi F-K^{2} \frac{2 n \beta^{2}}{1-\varepsilon} t^{2} \\
& =\frac{(1-\varepsilon)}{2 n \beta^{2}} F^{2} \psi^{2}-a \psi F t-\psi F \\
& -\left(\frac{n c}{R^{2}}+\frac{(n-1) C \sqrt{K}}{R}+2 \frac{C^{2}}{R^{2}}+\frac{n}{(1-\varepsilon)} \frac{C^{2}}{R^{2}}\right) F \psi t-K^{2} \frac{2 n \beta^{2}}{1-\varepsilon} t^{2} .
\end{aligned}
$$

Notice that here, we used the following inequality

$$
-\frac{2 F}{\beta} \frac{\nabla \psi \nabla W}{W} \geq-F \frac{|\nabla \psi|^{2}}{\psi} \frac{n}{(1-\varepsilon)}-\psi F \frac{|\nabla W|^{2}}{W^{2}} \frac{(1-\varepsilon)}{n \beta^{2}} .
$$

Thus, (3.22) implies

$$
\begin{aligned}
& \frac{|\nabla W|^{2}}{W^{2}}+\frac{b \beta^{2}}{\alpha} W^{\frac{1-\beta}{\alpha}}-\frac{a \beta}{\alpha} \ln W+\delta \frac{W_{t}}{W} \\
& =\beta^{2}\left(\frac{|\nabla u|^{2}}{u^{2}}+\frac{b}{\alpha} u^{\alpha-1}+\frac{a}{\alpha} \ln u-\frac{2}{\alpha} \frac{u_{t}}{u}\right)
\end{aligned}
$$




$$
\begin{aligned}
\leq & \frac{1}{t} \frac{2 n \beta^{2}}{(1-\varepsilon)}+\frac{2 n \beta^{2}}{(1-\varepsilon)}\left(\frac{n c}{R^{2}}+\frac{(n-1) C \sqrt{K}}{R}+\frac{2 C^{2}}{R^{2}}+a+\frac{n}{(1-\varepsilon)} \frac{C^{2}}{R^{2}}\right) \\
& +K \frac{2 n \beta^{2}}{1-\varepsilon} .
\end{aligned}
$$

Letting $\varepsilon$ sufficiently small, we obtain

$$
\begin{aligned}
& \frac{|\nabla u|^{2}}{u^{2}}+\frac{b}{\alpha} u^{\alpha-1}+\frac{a}{\alpha} \ln u-\frac{2}{\alpha} \frac{u_{t}}{u} \\
& \leq \frac{2 n}{t}+2 n\left(\frac{n c}{R^{2}}+\frac{(n-1) C \sqrt{K}}{R}+\frac{2 C^{2}}{R^{2}}+a+\frac{2 C^{2}}{R^{2}}+n \frac{C^{2}}{R^{2}}\right)+2 n K .
\end{aligned}
$$

Thus, we get the gradient estimate in Theorem B, which can be restated more precisely as follows:

Theorem 3.3 Let $M$ be an n-dimensional complete noncompact Riemannian manifold with Ricci curvature bounded below by $-K(K \geq 0)$. Assume $p \in M$ and $B_{p}(2 R)$, the geodesic ball of radius $2 R$ around $p, Q_{2 R, T}={ }^{\Delta} q B_{p}(2 R) \times[0, T]$. Suppose that $u(x, t)$ is a positive solution of (1.6) on $M \times[0, T]$ satisfying $\ln u \geq 0$. If $a \geq 0, b \geq 0$, and $0<\alpha<\frac{n}{n-1}$, then on $Q_{2 R, T}$, we have the following estimate for some constant $C>0$.

(I) If $0<\alpha<1$ or $1<\alpha<\frac{n}{n-1}$,

$$
\begin{aligned}
& \frac{|\nabla u|^{2}}{u^{2}}+\frac{b}{\alpha} u^{\alpha-1}+\frac{a}{\alpha} \ln u-\frac{2}{\alpha} \frac{u_{t}}{u} \\
& \leq \frac{1}{t} \frac{2 n}{\alpha^{2}}+\frac{2 n}{\alpha^{2}}\left(\frac{n c}{R^{2}}+\frac{(n-1) C \sqrt{K}}{R}+\frac{2 C^{2}}{R^{2}}+a\right. \\
& \left.\quad+\frac{2 C^{2}}{R^{2}}+\frac{n}{(2-\alpha) \alpha} \frac{C^{2}}{R^{2}}\right)+\left(K+\frac{a}{2}\left|\frac{1}{\alpha}-1\right|\right) \frac{2 n}{(2-\alpha) \alpha} .
\end{aligned}
$$

(II) If $\alpha=1$,

$$
\begin{aligned}
& \frac{|\nabla u|^{2}}{u^{2}}+\frac{b}{\alpha} u^{\alpha-1}+\frac{a}{\alpha} \ln u-\frac{2}{\alpha} \frac{u_{t}}{u} \\
& \leq \frac{2 n}{t}+2 n\left(\frac{n c}{R^{2}}+\frac{(n-1) C \sqrt{K}}{R}+\frac{2 C^{2}}{R^{2}}+a+\frac{2 C^{2}}{R^{2}}+n \frac{C^{2}}{R^{2}}\right)+2 n K .
\end{aligned}
$$

As $R \rightarrow+\infty$, we obtain the following 
Corollary 3.4 Let $M, u, a, b$, and $\alpha \in \mathbb{R}^{+}$be as in Theorem 3.3. Then,

$$
\begin{aligned}
& \frac{|\nabla u|^{2}}{u^{2}}+\frac{b}{\alpha} u^{\alpha-1}+\frac{a}{\alpha} \ln u-\frac{2}{\alpha} \frac{u_{t}}{u} \\
& \quad \leq \frac{1}{t} \frac{2 n}{\alpha^{2}}+\frac{2 n a}{\alpha^{2}}+\frac{2 n K}{(2-\alpha) \alpha}+\frac{n a}{(2-\alpha) \alpha}\left|\frac{1}{\alpha}-1\right|
\end{aligned}
$$

\section{Harnack Inequality}

In this section, we use the gradient estimates of the previous section to derive the corresponding Harnack inequality.

Theorem 4.1 Suppose that $M, u, a, b$, and $\alpha \in \mathbb{R}^{+}$are as in Corollary 3.4. Then,

$$
\begin{aligned}
u\left(x_{1}, t_{1}\right) \leq & u\left(x_{2}, t_{2}\right)\left(\frac{t_{2}}{t_{1}}\right)^{\frac{n}{\alpha}} \exp \left(\frac{\rho^{2}}{2\left(t_{2}-t_{1}\right) \alpha}+\frac{\left(t_{2}-t_{1}\right) n K}{(2-\alpha)}\right. \\
& \left.+n a\left(t_{2}-t_{1}\right)+\frac{n a}{2(2-\alpha)}\left|\frac{1}{\alpha}-1\right|\left(t_{2}-t_{1}\right)\right)
\end{aligned}
$$

where $x_{1}, x_{2} \in M, 0<t_{1}<t_{2}<\infty$, and $\rho\left(x_{1}, x_{2}\right)$ denote the geodesic distance between $x_{1}$ and $x_{2}$.

Proof If $f=\ln u$, then (3.25) can be rewritten as

$$
\begin{aligned}
|\nabla f|^{2}-\frac{2}{\alpha} f_{t} & \leq \frac{|\nabla u|^{2}}{u^{2}}+\frac{b}{\alpha} u^{\alpha-1}+\frac{a}{\alpha} \ln u-\frac{2}{\alpha} \frac{u_{t}}{u} \\
& \leq \frac{1}{t} \frac{2 n}{\alpha^{2}}+\frac{2 n a}{\alpha}+\frac{2 n K}{(2-\alpha) \alpha}+\frac{n a}{(2-\alpha) \alpha}\left|\frac{1}{\alpha}-1\right|
\end{aligned}
$$

Let $\left(x_{1}, t_{1}\right),\left(x_{2}, t_{2}\right) \in M \times[0, T]$ with $0<t_{1}<t_{2}$, and $\rho:[0,1] \rightarrow M$ be the minimal geodesic between $x_{1}$ and $x_{2}$ with $\rho(0)=x_{2}$ and $\rho(1)=x_{1}$. Define the curve $\eta:[0,1] \rightarrow M \times[0, T]$ by $\eta(s)=\left(\rho(s),(1-s) t_{2}+s t_{1}\right)$. Clearly, $\eta(0)=\left(x_{2}, t_{2}\right)$, $\eta(1)=\left(x_{1}, t_{1}\right)$ and

$$
\begin{aligned}
f\left(x_{1}, t_{1}\right)-f\left(x_{2}, t_{2}\right) & =\int_{0}^{1} \frac{d f(\eta(s))}{d s} d s \\
& \leq \int_{0}^{1}\left(\rho|\nabla f|-\left(t_{2}-t_{1}\right) f_{t}\right) d s .
\end{aligned}
$$

Using (4.2), we then have

$$
-f_{t} \leq-\frac{\alpha|\nabla f|^{2}}{2}+\frac{n}{\alpha t}+\frac{n K}{(2-\alpha)}+n a+\frac{n a}{2(2-\alpha)}\left|\frac{1}{\alpha}-1\right| .
$$


Thus, we have

$$
\begin{aligned}
f\left(x_{1}, t_{1}\right)-f\left(x_{2}, t_{2}\right) \leq & \int_{0}^{1}\left\{\rho|\nabla f|-\frac{\left(t_{2}-t_{1}\right) \alpha|\nabla f|^{2}}{2}+\frac{\left(t_{2}-t_{1}\right) n}{\alpha t}\right. \\
& \left.+\frac{\left(t_{2}-t_{1}\right) n K}{(2-\alpha)}+n a\left(t_{2}-t_{1}\right)+\frac{n a}{2(2-\alpha)}\left|\frac{1}{\alpha}-1\right|\left(t_{2}-t_{1}\right)\right\} d s,
\end{aligned}
$$

where $t=(1-s) t_{2}+s t_{1}$. Substituting the following inequality

$$
\rho|\nabla f|-\frac{\left(t_{2}-t_{1}\right) \alpha|\nabla f|^{2}}{2} \leq \frac{\rho^{2}\left(x_{1}, x_{2}\right)}{2\left(t_{2}-t_{1}\right) \alpha}
$$

into (4.3), one obtains

$$
\begin{aligned}
f\left(x_{1}, t_{1}\right)-f\left(x_{2}, t_{2}\right) \leq & \frac{\rho^{2}}{2\left(t_{2}-t_{1}\right) \alpha}+\left(\frac{n}{\alpha}\right) \ln \left(\frac{t_{2}}{t_{1}}\right)+\frac{\left(t_{2}-t_{1}\right) n K}{(2-\alpha)} \\
& +n a\left(t_{2}-t_{1}\right)+\frac{n a}{2(2-\alpha)}\left|\frac{1}{\alpha}-1\right|\left(t_{2}-t_{1}\right),
\end{aligned}
$$

hence,

$$
\begin{aligned}
u\left(x_{1}, t_{1}\right) \leq & u\left(x_{2}, t_{2}\right)\left(\frac{t_{2}}{t_{1}}\right)^{\frac{n}{\alpha}} u \exp \left(\frac{\rho^{2}}{2\left(t_{2}-t_{1}\right) \alpha}+\frac{\left(t_{2}-t_{1}\right) n K}{(2-\alpha)}\right. \\
& \left.+n a\left(t_{2}-t_{1}\right)+\frac{n a}{2(2-\alpha)}\left|\frac{1}{\alpha}-1\right|\left(t_{2}-t_{1}\right)\right) .
\end{aligned}
$$

This finishes the proof of Theorem 4.1.

Acknowledgments Supported partially by NSF of China (No. 11171253).

\section{References}

1. Calabi, E.: An extension of E. Hopf's maximum principle with an application to Riemannian goemetry. Duke Math. J. 25, 45-56 (1958)

2. Cheng, S.Y., Yau, S.T.: Differential equations on Riemannian manifolds and their geometric applications. Commun. Pure Appl. Math. 28, 333-354 (1975)

3. Gidas, B., Spruck, J.: Global and local behavior of positive solutions of nonlinear elliptic equations. Commun. Pure Appl. Math. 34, 525-598 (1981)

4. Hamilton, R.: Three-manifolds with positive Ricci curvature. J. Differ. Geom. 17, 255-306 (1982)

5. Li, P., Yau, S.T.: On the parabolic kernel of the Schrodinger operator. Acta Math. 156, 153-201 (1986)

6. Li, J.: Gradient estimate and Harnack inequalities for nonlinear parabolic and nonlinear elliptic equations on Riemannian manifolds. J. Funct. Anal. 100, 233-256 (1991)

7. Ma, L.: Gradient estimates for a simple elliptic equation on complete non-compact manifolds. J. Funct. Anal. 241, 374-382 (2006)

8. Yang, Y.: Gradient estimate for a nonlinear parabolic equation on Riemannian manifold. Proc. Am. Math. Soc. 136, 4095-4102 (2008) 